\title{
Messapico Messapian
}

\author{
Simona Marchesini \\ Alteritas - Interazione tra i popoli \\ s.marchesini@alteritas.it
}

Riassunto: Il Messapico è la lingua preromana di frammentaria attestazione della II regione augustea Apulia et Calabria, attestata tra il VI ed il II sec. a.C. Sono note ca. 650 iscrizioni, di contenuto prevalentemente onomastico. La posizione linguistica del Messapico è ancor oggi dibattuta, perché questa lingua, pur appartenendo alla famiglia Indo-Europea, non appare affine ad alcuna delle altre lingue dell'Italia antica, classificandosi dunque come 'isolata'. L'A., dopo una panoramica sulla Puglia nell'antichità come presentata dalle fonti storiche $\mathrm{e}$ sulla definizione del termine 'Messapico', descrive gli aspetti epigrafici e linguistici, con un approfondimento sul tema della palatalizzazione messapica e del genitivo -ihi, presentando il quadro delle varie posizioni ermeneutiche.

Parole chiave: Messapico. Puglia. Linguistica indoeuropea. Lingue frammentarie. Epigrafia preromana.

Abstract: Messapian is the pre-Roman fragmentary language of the $2^{\text {nd }}$ Augustan region Apulia et Calabria, attested between the $6^{\text {th }}$ and the $2^{\text {nd }} \mathrm{c}$. BC. Almost 650 inscriptions, mostly personal names, are known from this region. The linguistic position of the language within the IndoEuropen language is still debated, as it seems to be isolated in the framework of the languages known in ancient Italy. The author offers an overview of pre-Roman Apulia as described by ancient authors, focussing on the definition of the term 'Messapic'. She further describes the epigraphic and linguistic features, with in-depth considerations on the Messapic palatalisation and on the -ihi genitive, topics which still raise discussion among the specialistst.

Keywords: Messapic. Apulia. Indo-European linguistics. Relic languages. Pre-Roman epigraphy.

Recepción: 10.09.2019 | Aceptación: 11.03.2020 


\section{Introduzione e stato dell'arte}

\subsection{Definizione e classificazione linguistica}

Con Messapico si intende la lingua parlata nella antica Puglia, corrispondente alla Regio II Apulia et Calabria. Gli autori greci antichi, tra questi in modo più specifico Nicandro fr. 47 Schneider, apud Antoninus Liberalis, Metamorphoses 31 (età ellenistica), ma anche prima di lui Erodoto Hist. 4.99.2-5 (VI sec. a.C.) e lo Pseudo Scilace Per. 14 (IV sec. a.C.), designavano con Iapigia

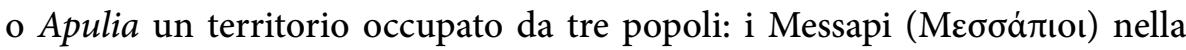
zona meridionale a sud della linea che collega Taranto a Brindisi fino a Leuca,

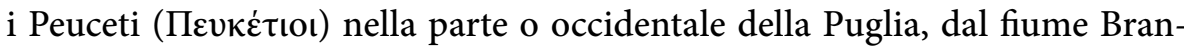
dano fino alle Murge e al fiume Ofanto, e i Dauni ( $\Delta$ av́vıoı) più a nord, nella penisola del Gargano. La definizione viene ripresa anche da Polibio 3.88.4 in età augustea. I dati archeologici, seppure stratificati tra loro, confermano almeno in parte questo dato, come evidenziato soprattutto dalla diffusione dei cd. vasi Apuli nel V e IV secolo a.C. (Lombardo 2014).

Una descrizione un po' diversa viene offerta da fonti di età tardo repubblicana. Strabone Geog. 6.3.1, C277 (età augustea) ricorda che la differenziazione di questi popoli con le relative denominazioni è un aspetto suggerito dalle fonti greche, ma che in realtà gli autoctoni chiamavano sé stessi con altri nomi. Ad esempio, nella parte meridionale dell'Apulia essi tenevano distinti i Salentini ( $\Sigma \alpha \lambda \varepsilon v \tau i v o l)$, che occupavano la parte meridionale della penisola,

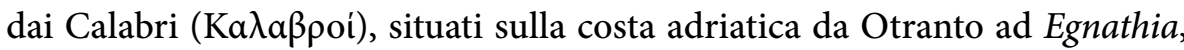
con il loro hinterland. Secondo Strabone i popoli di questarea, pur chiaman-

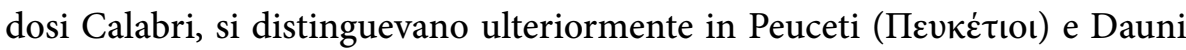
( $\Delta$ av́vıoı), ma in definitiva la regione settentrionale nel suo insieme veniva chiamata Apulia. Questa situazione, testimoniata anche da Plinio il Vecchio Nat. 3.99.102-104 (I sec. d.C.), ci restituisce un quadro stratificato e variegato dell'effettivo popolamento delle antiche popolazioni nella antica Apulia (per una raccolta sistematica delle fonti antiche $c f$. Lombardo 1992), di cui gli etnonimi costituiscono un riflesso. 


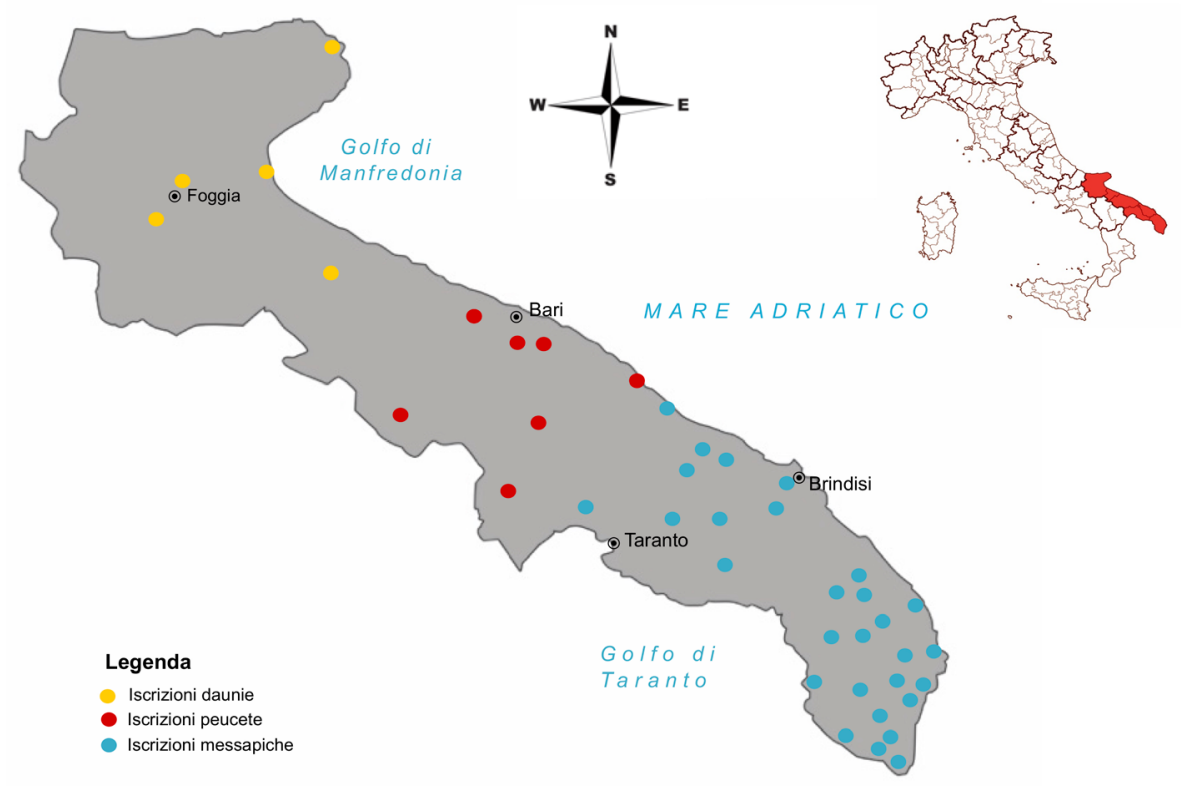

$\overline{\text { Fig. 1. Puglia e distribuzione della documentazione epigrafica. }}$

Se verifichiamo questi dati con la situazione epigrafica e linguistica, vediamo che effettivamente una differenza sostanziale è ravvisabile tra la documentazione messapica vera e propria, a cui vanno riferiti anche i documenti provenienti da Ostuni, Ceglie, Carovigno e Gnathia - quindi poco al di sopra della linea che collega Brindisi e Taranto - e la zona settentrionale, designata come Peuceta e Daunia. Cè da dire che dalla zona Peuceta provengono pochi documenti, che difficilmente consentono un inquadramento epigrafico e linguistico. In particolare, ad Adelfia, Azetium (Rutigliano) e Massafra, il cattivo stato di conservazione o lesiguità dei documenti epigrafici non consentono di riconoscere dei tratti epigrafici ben definiti.

In ambito daunio invece i documenti, che iniziano con il IV secolo e si attestano fino alla romanizzazione, sono più numerosi e in alcuni casi più lunghi. In essi è possibile il riconoscimento di varietà grafiche, da valutare come esito di tratti fonologici effettivamente differenziati rispetto al Messapico.

Nell'insieme l'epigrafia messapica costituisce un piccolo ma importante tassello nellambito dell'area linguistica Indo-Europea e del Mediterraneo 
antico, caratterizzato dall'isolamento rispetto agli altri rami della famiglia linguistica indo-europea.

Da un punto di vista quantitativo, il Messapico copre, con le sue ca. 600 iscrizioni conosciute fino ad oggi, una posizione intermedia nelle lingue frammentarie dell'Italia Preromana, leggermente superiore al Retico (350 iscrizioni $c a$.) al Venetico (300 iscrizioni $c a$.), e inferiore alle attestazioni del gruppo sabellico, che si attestano sulle 800 unità.

L'evidenza epigrafica del santuario marittimo di Grotta della Poesia Piccola, nel Salento (Roca Vecchia, Melendugno, LE), con le sue pareti aggettanti iscritte in iscrizioni sovrapposte in molti strati su una superficie di ca. $600 \mathrm{mq}$, è destinata a far aumentare sensibilmente il numero delle iscrizioni e quindi anche il quadro epigrafico e linguistico del Messapico. A questo proposito, un consorzio costituito da nove partner tra istituzioni locali ed enti di ricerca no-profit si è costituito nel 2017 per lo studio, la valorizzazione e la conservazione del complesso di Grotta Poesia.

Dalle fotogrammetrie compiute sui calchi creati negli anni '90 da C. Pagliara per conto dell'Università del Salento, attualmente conservati presso il Dipartimento di Scienze dell'Antichità della stessa università, risulta che a testi di tipo devozionale, nel complesso ben leggibili, si affianca nella grotta la fitta rete di iscrizioni sovrapposte e illeggibili. Un confronto antropologico ed etnografico induce a pensare (Marchesini c.s. 1; c.s. 2) che questi testi si riferiscano ad "ego inscriptions", ad una classe di testi frequenti in luoghi speciali come i santuari. Se così fosse, il contenuto delle iscrizioni avvolte nella fitta rete di grafi sarebbe costituito per lo più da nomi personali, date e altri enunciati brevi, riducendo così la nostra speranza di guadagnare sensibili novità sugli aspetti grammaticali della lingua messapica.

Per quanto riguarda la classificazione del Messapico come lingua indoeuropea non si è trovato ancora un accordo generale sulla sua pertinenza ad un sottogruppo linguistico specifico all'interno della famiglia linguistica. Il comportamento apparentemente contraddittorio di alcuni esiti indoeuropei rende difficile lattribuzione del Messapico alle lingue del gruppo centum o a quelle "orientali" del gruppo satem. Così ad esempio la vocale indoeuropea / in particolare in quelle dell'Italia antica, è trattata in messapico come /ă/.

Essendo l'Apulia sostanzialmente una penisola (vedi fig. 1), le sole lingue confinanti sono i dialetti oschi attestati presso i suoi confini settentrionali e 
nord-orientali. Qui le comunità, conosciute dalle testimonianze latine e dalla cultura materiale ed epigrafica, scrivevano con un alfabeto derivato da quello etrusco, come i Frentani, i Larinates, gli Hirpini (a nord), altri con alfabeto di tipo greco, come i Lucani ed infine i Brettii più a sud (Marchesini 2009, tav. X; si vedano anche Bourdin 2012; Farney e Bradley 2017; Benelli, Marchesini e Poccetti 2020). In tutti i casi le testimonianze epigrafiche di questi popoli italici si datano a partire sostanzialmente dal IV secolo, quindi in un periodo più tardo rispetto all'inizio dell'epigrafia messapica all'inizio del VI. Le condizioni geografiche, che vedono la Messapia più aperta verso il mare che verso l'entroterra italico, da cui la divide anche l'Appennino, hanno probabilmente determinato una scarsa osmosi verso gli altri popoli confinanti, a differenza di quanto avviene nel resto dell'Italia. Non vi sono infatti molti casi di scambi onomastici o imprestiti linguistici nelle due direzioni linguistiche, come si osservano invece nell'Italia centrale.

Gli imprestiti linguistici finora osservati in messapico sono pochi: ad esempio l'appellativo bennarrihi in genitivo, nell'iscrizione MLM 2 Os da Ostuni, per cui si $c f$. gall. benna; lat. bennarius (MLM II, s.v.), oppure argora-pandes, in cui il primo elemento, 'denaro', trova il suo diretto confronto in à $\rho$ voos, mentre il secondo, ${ }^{*}$-pandyas $\left(<{ }^{*}\right.$-pondyos) trova confronto con il latino pendō ('pagare', 'stimare': l'equivalente sarebbe *argento-pondius; $c f$. per la formazione il gr. á $\rho \gamma v \rho o-\sigma \tau \alpha \dot{\tau \eta} \varsigma$ in Aristofane; si veda anche il gallico arganto-dan- 'tesoriere': MLM II, s. v.). In questi casi però non è esclusa una comune discendenza dalla lingua indo-europea per i due esiti. Un altro caso di imprestito viene dal Latino calator, ovvero il nome Kalatoras, in genitivo, usato come gentilizio in una formula onomastica da Taranto (MLM $1 \mathrm{Ta}$, seconda metà III-fine II sec. a.C.).

Poco differente è la situazione nei nomi personali, in cui si individuano solo pochi casi di imprestiti onomastici. Dall'area osca proviene il prenome maschile Mahehos di un'iscrizione da Ceglie (ora dispersa, MLM $28 \mathrm{Cae}$, III sec. a.C.) che compare in formula onomastica con Verrinihi, genitivo di Verrines, di chiara ascendenza latina ( $c f$. il gentilizio Verrinius, Solin e Salomies 1988, 203). In un'iscrizione da Aletium troviamo la variante Mahehas (per -o-? MLM $53 \mathrm{Al}$ ) o ancora troviamo Mehehe (MLM 2 Car da Carovigno, IV sec. a.C.) la cui origine italica è assicurata dalla presenza in ambito sabellico del PN Maís gentilizio Mahiis (cf. Lejeune 1975, 88, 110; $c f$. anche Rix, ST, 141).

Un caso di nomi personali imprestati dal latino si trova nell'iscrizione MLM 1 Di da Diso (III sec. a.C.), in cui la formula femminile [s?]ekonda ke- 
zareihei costituisce ladattamento in messapico di due nomi latini, con resa fonologica di /u/ in /o/ in Sekonda e con il genitivo del nome aggiunto in -eihei. Si tratta in questo caso dell'integrazione di una donna latina o di discendenza latina nella compagine messapica (cf. Däuber 1991, 327-333 e da ultimo Marchesini c.s. 1). Il fatto - peraltro raro - che la donna sia il soggetto di una dedica scritta su pietra ci fa pensare che il suo livello sociale sia elevato. $\grave{E}$ bene ricordare qui che l'evidenza onomastica restituisce invece un quadro in cui il gentilizio è diffuso soprattutto nella compagine maschile della società messapica, come individuato da J. Untermann 1964.

Un altro possibile imprestito dal mondo latino è l'appositivo (gentilizio) Koileih[i?], dal gentilizio latino Coelius su un'iscrizione da Rudiae (MLM3 Rud, III sec. a.C.). Anche in questo caso il nome è integrato morfologicamente nel repertorio messapico (genitivo in -ihi). Ancora il gentilizio maschile $\operatorname{Kor}(\theta) i h i$ (MLM 1 Lup, da Lupiae, III sec. a.C.) o KorHeihi (MLM 10 Uz, da Ugento, metà III-fine II sec. a.C.) in genitivo, potrebbero rappresentare la forma messapica del nome latino Curtius (MLM II, s.v.). Analogamente il Kokkeias dell'iscrizione sulla parete di Grotta Poesia (Melendugno, LE), gentilizio maschile in nominativo in un'iscrizione votiva a Venas e @autour Andiraho - sempre che la lettura venga confermata dalle indagini in corso - , potrebbe corrispondere al gentilizio latino Cocceius. In questo caso l'orizzonte cronologico è incerto, ma le iscrizioni finora identificate sembrano essere inquadrabili dopo il IV secolo a.C. Anche il gentilizio Megones sempre da Grotta Poesia (MLM 23 Ro) appare come un imprestito dal latino-italico Megonius (Schulze 1904, 153).

Come imprestiti del Messapico in altre lingue si possono citare i casi onomastici di Dasio, Dasius, gentilizi latini derivati molto probabilmente dal comune prenome messapico Dazes, diffusi anche in area illirica (ZGLE, 31, $38,39,44,287,423,519)$. Anche il gentilizio lat. Barrius, attestato a Taranto, o il nome personale greco Báppıs (II sec. a.C.) sembrano provenire da area Messapica, dove il gentilizio Barreinihi (MLM 19 Rud) dimostra lesistenza di una base onomastica Barres (MLM II, s. v.).

Nel complesso, lo scambio con i popoli prossimi di ambito italico appare limitato, mentre più consistente si segnala, in età di romanizzazione a partire dal III sec. a.C., l'integrazione di cittadini romani nel contesto sociale messapico.

Per quanto riguarda i Greci italioti, provenienti dalle colonie, essi lasciano le loro tracce non solo nella produzione di vasi apuli, per i quali sono state riconosciute maestranze greche coloniali operanti nei laboratori dei centri 
Dauni e Peuceti a fianco di artigiani locali, ${ }^{1}$ ma anche tracce epigrafiche, come ad esempio il vaso da Azetium (Rutigliano, in Peucetia) con iscrizione dipinta in cui per esprimere un nome locale si usa l'alfabeto greco $\triangle \mathrm{A} \Sigma \mathrm{TA} \Sigma \mathrm{EMI}$ oppure la lekythos apula da Gnathia con l'iscrizione $\Gamma$ PAIA $\Sigma$, del 400-350 a.C. o ancora le due patere di IV secolo da Ruvo, con iscrizioni IK $\Omega$ e IKK $\Omega$, o infine la piramidetta fittile (peso da telaio) da Canosa, con iscrizione $\mathrm{B} \Lambda \mathrm{A}$ KAIFEIN YN (per tutti i casi si veda Marchesini 2013a, 27-28).

Messapi fuori dalla Messapia sono attestati dalle fonti storiche (si pensi al reclutamento di mercenari Iapigi, Peucezi e Messapici nella spedizione ateniese in Sicilia del 413 a.C., sui cui riferisce Tucidide, VII 133), ma anche dalla presenza del nome messapico $\triangle \mathrm{AZIMO}$ su un'anfora a enchytrismos usata per la sepoltura infantile di fine VII-inizi VI secolo a.C. da Pithekoussai (Marchesini 2013a, 29; cf. Lombardo 2014, 43-46; Boffa 2015, 9). Il nome, tra l'altro, è attestato anche in Acarnania $(\Delta \dot{\alpha} \zeta \mu \circ \varsigma)$ in area illirica (a Thyrreion nel II sec. a.C.: IG IX, $\mathrm{I}^{2} 2$ 247, 13; 248, 12-13; 250, 9 18; 252, 2-3) a Delo (Boffa 2015, 9).

Nell'insieme però sembra che non vi sia tra la Puglia e i territori confinanti delle culture italiche quell'osmosi culturale che si osserva nel resto dell'Italia centro-meridionale, fatta di scambi e condivisione di pratiche cultuali, repertori e sistema onomastico ( $c f$. Marchesini 2007, 117-130; $c f$. anche Bourdin 2012; Farney e Bradley 2017). I popoli che abitarono l'antica Puglia mantengono, sia in virtù della loro situazione geografica sia probabilmente della loro genesi etnografica, caratteristiche culturali e linguistico-epigrafiche proprie e ben definite.

\subsection{Localizzazione e cronologia delle iscrizioni}

La maggior parte delle iscrizioni messapiche proviene dalle necropoli di insediamenti urbani, come risultato di uno sviluppo demografico e sociale che portò al mutamento dei costumi funerari e alla monumentalizzazione delle tombe nel corso del VI secolo. I primi centri a presentare questo sviluppo sono Oria, Mesagne, Muro Tenente e Valesio; più tardi anche Manduria, Rudiae, Cavallino, Alezio, Ugento e Vaste si dotano di necropoli con tombe monumentalizzate iscritte (Lombardo 1994; 2013b).

Come detto sopra, la parte di Apulia che ha restituito più iscrizioni è quella meridionale, considerata propriamente messapica, mentre l'epigrafia

1 Si vedano a questo proposito Gadaleta 2012, 77-109; Todisco 2012; 2013, 343-345; Marchesini 2013a, 26-28. 
settentrionale in aree peuceta e daunia è ridotta e più tarda. Se infatti le più antiche iscrizioni messapiche si datano alla prima metà del VI secolo a.C., nel distretto settentrionale, a Ruvo e ad Arpi, le iscrizioni più antiche sono del IV secolo. A partire dal II sec. a.C., dopo la fase di massima produzione epigrafica del III secolo, si comincia a notare una riduzione del numero di epigrafi, che non vanno oltre l'inizio del II secolo a.C. Il processo di romanizzazione della Puglia, che comincia nel III secolo e si conclude con la lex Iulia municipalis nel 45 a.C. determina il progressivo abbandono della pratica epigrafica in lingua epicoria.

\subsection{Storiografia e stato dell'arte. Principale letteratura linguistica; corpora epigrafici}

La storia degli studi sul Messapico inizia con le prime pubblicazioni di T. Mommsen, che nel 1850 inserì le iscrizioni messapiche nel suo Unteritalische Dialekten. A partire dal 1881 W. Deecke iniziò a pubblicare sul Rheinisches Museum für Philologie una serie di contributi sulla lingua messapica, commentando anche nuove iscrizioni (Deecke 1881; 1887). H. Krahe dedicò alla lingua messapica una serie di contributi a partire dalla fine degli anni '20 (Krahe 1928; 1929). Nel 1933 R. S. Conway pubblicò, assieme a J. Whatmough e S. E. Johnson, I Prae Italic Dialects of Italy (PID).

Le prime opere sul Messapico soffrivano della mancanza di un adeguato corredo (foto)grafico, essendo la maggior parte dei disegni di iscrizioni fortemente interpretativi. Sempre a partire dagli anni '20 F. Ribezzo presentò un resoconto di tutto ciò che era stato pubblicato fino ad allora nella rivista Indo Greco Italica (dal 1922 al 1935), in una serie di contributi che poi confluirono nel Corpus Inscriptionum Messapicarum (CIM), edito da C. Santoro nel 1978. Il CIM costituisce senza dubbio un primo approccio scientifico allo studio della lingua messapica a partire dall'evidenza documentaria epigrafica. Anche in questo caso però la documentazione fotografica fornita non consentiva una lettura adeguata delle iscrizioni e Ribezzo proponeva per i testi isolati etimologie non sempre cogenti.

Limiti analoghi presenta la successiva opera di O. Parlangèli NIM 1-3, 1965-68, che presenta ancora un'edizione dei testi messapici senza adeguata documentazione fotografica o grafica. Nel 1955 e nel 1964 H. Krahe curò un'opera in due volumi sulla lingua degli Illiri (Die Sprache der Illyrier), che vedeva raccolti nel volume II due diversi saggi sulla lingua messapica, ad opera dei suoi allievi. C. de Simone presentò nella prima parte del volume 
(fino a p. 151) Die Messapische Inschriften, un corpus aggiornato di tutte le iscrizioni messapiche con adeguato apparato fotografico e, per la prima volta, un ordinamento cronologico di tutti i documenti in un grafico di seriazione epigrafica. La metodologia era improntata alle seriazioni cronologiche in uso nelle indagini preistoriche e paleontologiche e consentiva di uscire dal vicolo cieco di datazioni circolari, basando la cronologia su dati archeologici e contestuali esterni all'iscrizione stessa.

La seconda parte del volume, ad opera di J. Untermann, studiava il repertorio onomastico messapico e ne delineava le caratteristiche salienti, ancor oggi valide (Untermann 1964; De Simone 2013; si $c f$. infra).

Negli anni dal 1976, 1982 e 1984 C. Santoro pubblicò tre volumi dedicati alle iscrizioni messapiche, aggiungendo nuovi dati e presentando un buon corredo fotografico di ogni testo. Nel 2002 uscì il corpus Monumenta Linguae Messapicae, in due volumi, curato da C. de Simone e S. Marchesini. Il primo volume presenta le iscrizioni, corredate da foto ottenute in buona parte da nuove campagne di ricognizione, e apografi, con schede epigrafiche analitiche. Una breve introduzione ad ogni sito precede ogni sezione ordinata alfabeticamente per luogo, con dati quantitativi sui reperti e sul loro ritrovamento. Ogni scheda contiene, in forma distinta, la lettura diplomatica e quella interpretativa dei testi proposta dagli editori. In quest'opera il metodo della seriazione epigrafica viene reso più efficace dall'utilizzo di strumenti informatici, che hanno consentito di processare 506 iscrizioni e di inserirle nelle singole fasi epigrafiche ( $c f$. infra), datate per mezzo di alcuni oggetti significativi. Il volume II presenta le schede lessicali, che descrivono ogni voce isolata e riassumono tutta la bibliografia precedente e, dove possibile, offrono ricostruzioni etimologiche e inquadramenti semantici.

Dopo il 2002 solo pochi altri testi sono stati rinvenuti e pubblicati: 47 brevi testi su ceramica provenienti dall'area antistante della Grotta di Santa Maria di Agnano a Ostuni (Brindisi) con nomi di persona (Poccetti 2008), datati tra IV e III sec. a.C., due blocchi di calcarenite dall'Athenaion di Castrum Minervae del IV sec. a.C. (Lecce: D’Andria e Lombardo 2009), altri due testi, su ceramica e su pietra, da un luogo di culto a Vaste, datati tra IV e III sec. a.C. (Mastronuzzi e Ciuchini 2011) e un testo su una coppa da Conversano (Bari: Ferrandini Troisi 2005; per un aggiornamento Marchesini 2015). Tre coppe su alto piede greco-peucete da collezione privata, da Adelfia e da Valenzano (Peucezia), con sigla sovradipinta alz sono state attribuite ad epigrafia messapica per tipologia di lettere e per nome, che sembra abbreviazione di alz[an], 
o alz[enas] (attestati in messapico in $M L M 48$ Lup), ma stupisce l'antichità della documentazione alla prima metà VI-prima metà V sec. a.C., dato che l'epigrafia daunia e peuceta inizia almeno un secolo più tardi (Ferrandini Troisi 1992a, 216 e 1992b, 55-57; Ambrosini 2010-2013).

Da un punto di vista strettamente linguistico sono molti i contributi allo studio della flessione nominale messapica, soprattutto ad opera di Carlo de Simone 1978, 1982, 1983, 1988, 1992, 2012, 2013, Martin Huld 1995 Aldo Luigi Prosdocimi 1989, 1990, 2006, Roberto Gusmani 2006, Vincenzo Orioles 1991 e Marco Ciceri 2012. Hanno attratto l'attenzione degli studiosi anche gli studi sulla palatalizzazione, per i quali si veda infra, $\$ 1.4 .1$.

\subsection{La lingua. Fonetica, morfologia ed elementi sintattici, lexicon}

La lingua messapica appartiene alla famiglia linguistica indo-europea e come tale è di tipo flessivo.

È difficile definire con quale gruppo linguistico all'interno di questa famiglia il Messapico presenti i suoi più stretti legami. I tratti finora descritti impediscono una decisa attribuzione sia al gruppo occidentale delle lingue cd. centum, cui la lingua sembra appartenere per condizioni geografiche, sia al gruppo satem orientale, con cui invece sembra condividere alcuni aspetti come ad esempio una marcata palatalizzazione, peraltro osservata fino ad oggi sostanzialmente nei nomi di persona. Il repertorio lessicale offerto dalle iscrizioni è assai scarso, essendo costituita la maggioranza dei testi da nomi personali. Ricordiamo qui che la "grammatica" dei nomi personali è strutturalmente (ma anche neurologicamente) distinta da quella dei nomi comuni (sul diverso trattamento linguistico dei propria si veda Marchesini 2007, 17-23). I nomi propri tendono ad essere conservativi rispetto allo sviluppo linguistico degli appellativa, per cui considerare sviluppi fonologici o morfologici a partire dai propria potrebbe essere fuorviante per considerazioni sulla diacronia linguistica.

Detto questo, il Messapico presenta aspetti contraddittori rispetto al trattamento di tratti fonologici, come ad esempio la restituzione della vocale i.e. $I^{*} \breve{\mathrm{o}} /$, resa con ${ }^{*} \breve{\mathrm{a}} /$ ( $^{*}$ to-bhoros, da ${ }^{\star} b^{h}$ er- 'portare' > tabara 'sacerdotessa'). Tutte le altre lingue del gruppo occidentale, infatti, comprese il Greco e le lingue dell'Italia antica, conservano la vocale originaria. Il più diretto confronto per tale mutamento è il Sanscrito, che però appartiene al gruppo satem. 
Un altro fenomeno macroscopico che caratterizza il Messapico è la palatalizzazione, già oggetto di studio a partire da V. Pisani, che individuava in questo fenomeno un "riassetto del (dia)sistema consonantico italico" che interessò molte lingue dell'Italia antica, sia indoeuropee che non (ma si veda il $\S$ seguente).

\subsubsection{Fonetica/fonologia}

L'inventario dei segni messapici corrisponde sostanzialmente a quello dell'alfabeto laconico di Taranto. Come spesso avviene, nell'acquisizione di un alfabeto si operano sempre quelle modifiche di adattamento che prevedono eliminazione, addizione e modificazione dei segni. Questi interventi consentono l'adattamento del set alfabetico originario alle esigenze della lingua ricevente. Anche il Messapico ha operato, dopo una fase iniziale di sperimentazione, le modifiche di adattamento dell'inventario dei grafemi. All'inizio dell'esperienza epigrafica si osservano componenti di altri alfabeti, come quello corinzio o euboico (il beta con occhielli angolati, assente nell'alfabeto tarantino, l'alpha arrotondato di tipo euboico: si veda Marchesini 1999, 202).

La riforma operata dopo il periodo di sperimentazione vede l'eliminazione del grafema $\langle\varphi\rangle$ : il suono corrispondente viene restituito con il grafema della sorda $<\mathrm{p}>$ (es. Aprodita).

Il grafema a tridente $\Psi$, con le varianti $\Psi, \uparrow$ viene impiegato per rendere una fricativa che potrebbe essere vicina ad $/ \mathrm{h} /$, a giudicare dalle scritture come HaivaYias (da *hava-ya-s?) di MLM 12 Bal, da Valesio (prima metà VI-prima metà V sec. a.C.) oppure Bale ias (ci aspetteremmo ${ }^{\star}$ Balehias; MLM $15 \mathrm{Bal}$, da Valesio, stessa cronologia del precedente). Ulteriore prova è il fatto che nel III secolo la scrittura haivahias (MLM 3 Car) reinserisce la $<\mathrm{h}>$ al posto del precedente segno a tridente.

Il segno a tridente a base quadrata $\langle\Psi\rangle$ invece, usato talvolta al posto del $\langle\theta\rangle$, esprime una aspirata sorda, e sembra alternare con il grafema theta, come mostrano le scritture Aaotoras/ $\Psi$ aotoras.

Un altro grafema inserito ex-novo rispetto alla serie laconico-tarantina è il segno croce di S. Andrea $\langle\mathrm{X}\rangle$, che ha come variante il segno a croce greca $<+>$. Se negli alfabeti greci occidentali il segno rendeva una fricativa $/ \mathrm{ks} /$, in Messapico viene usato per una spirante palatale /š/. 
Il grafema $\langle\theta\rangle$ viene utilizzato in messapico soprattutto in inizio di parola per l'aspirata dentale sorda $/ \theta /$, ma nel digrafo $\langle\mathrm{t} \theta>$, il theta rende la palatalizzazione della dentale a contatto con $/ \mathrm{y} /$, come in blat $\theta e s$ ed et $\theta e t a$.

Infine, il grafema $<\mathrm{z}>$ viene usato in inizio di parola sia come esito di ${ }^{\star} d y$ (zis $<{ }^{\star}$ dyes) sia come spirante dentale sonora $/ \mathrm{z} /, \mathrm{e}<\mathrm{sz}>$ : daszes come esito di palatalizzazione $\left({ }^{*} d a z-y a-s\right)$.

La lingua messapica sembra aver distinto tra vocali brevi e lunghe, come si evince dalle scritture vaanetos, kraapati, soolles, o come bohonihi, mooklioos, che presentano un allungamento della vocale mediante il suo raddoppiamento o mediante inserzione del grafema $<\mathrm{h}>$.

Come elementi caratterizzanti la fonologia del Messapico possiamo ricordare, come detto sopra, la resa di i.e. / ${ }^{\star}$ o/ come /a/, evidente in tabara ('sacerdotessa') da ${ }^{\star}$ to-bhoros. Ne deriva che il grafema $<0<$ in messapico, quando attestato, è da intendere come una $<\overline{\mathbf{o}}>$, realizzazione di $/ \mathrm{u} /$.

Il Messapico presenta qui una differenziazione tra scritture meridionali (propriamente messapiche) e quelle settentrionali (daunie) proprio per la realizzazione di questo fonema, che è realizzato nelle iscrizioni daunie con $\mathrm{i}$ grafemi $<$ y $>$ e $<$ v $>$.

Il sistema vocalico della lingua messapica risulta nel complesso asimmetrico, avendo un solo grafema per la serie velare, rispettivamente $<0>$ in messapico proprio $\mathrm{e}<\mathrm{y}>,<\mathrm{v}>$ per la variante daunia, tanto che viene usato per questo fonema il termine di "arcifonema"/ü/, ovvero un suono che assume su di sé tutta la serie delle vocali velari.

Un altro tratto che contraddistingue la differenza tra varianti settentrionali e meridionali è loscillazione tra /i/ e /o/ nella variante meridionale e /i/ e /u/ nella variante apula. Scritture come Damatira/Damatura (Daunio) o come Dazimas/Dazomas (messapico) presentano gli effetti grafematici di una neutralizzazione dellarcifonema /ü/ a contatto con labiali. Il fenomeno è altrettanto presente in latino, dove troviamo forme come optumus accanto a optimus (Leumann 1972,88) o nelle lingue sabelliche, dove ad esempio il marrucino vede l'alternanza di cibat/cubat (Untermann 2000, 406).

Un'oscillazione tra /e/ ed /a/ in alcuni nomi, come kezareihei (MLM 1 Di, da Diso) vs. kazarei (MLM 1 Bas da Vaste), entrambe di III sec. a.C., può essere effetto di una palatalizzazione progressiva esercitata dalla consonante precedente (De Simone 1992, 7; Matzinger 2019, 26). 
Il sistema consonantico, riassunto nella tabella che segue (Tab.1), attesta tra le occlusive le sorde e le sonore, ma non le aspirate. Per le labiali abbiamo infatti /p/ e /b/, per le dentali /t/ e /d/, per le velari /g/ e /k/. Le affricate sono rappresentate dalle sonore $/ \mathrm{dz} / \mathrm{e} / \mathrm{ts} /$. Si conoscono inoltre tra le spiranti la palatale sonora /ž/, la dentale sorda /s/ e la palatale sorda /š/.

\begin{tabular}{|c|c|c|c|c|c|}
\hline Occlusive & Labiali & Dentali & Velari & Palatali & Glottali (?) \\
\hline Sonore & $/ \mathrm{b} /(<\mathrm{b}>)$ & $/ \mathrm{d} /(<\mathrm{d}>)$ & $/ g /(<g>)$ & & \\
\hline Sorde & $/ \mathrm{p} /(<\mathrm{p}>)$ & $/ \mathrm{t} /(<\mathrm{t}>)$ & $/ \mathrm{k} /(<\mathrm{k}>)$ & & \\
\hline \multicolumn{6}{|l|}{ Affricate } \\
\hline Sonore & & $/ \mathrm{ds} /(<\mathrm{z}>)$ & & & \\
\hline Sorde & & $/ \mathrm{ts} /(<\theta>)$ & & & \\
\hline \multicolumn{6}{|c|}{ Spiranti/Fricative } \\
\hline Sonore & & - & & $\mid \check{z} /(<\mathrm{Z}>)$ & \\
\hline Sorde & & $/ \mathrm{s} /(<\mathrm{s}>)$ & & $/ \check{s} /(<+>)$ & \\
\hline \multicolumn{6}{|l|}{ Aspirate } \\
\hline Sonore? & & & & & $/ \mathrm{h} /(?)(<\mathrm{h}>)$ \\
\hline
\end{tabular}

Tab. 1. Il sistema delle consonanti.

Per quanto riguarda il trattamento delle medie aspirate, che come noto contraddistingue le lingue italiche (cf. Stuart-Smith 2004; Clackson 2013, 2), il Messapico non sembra presentare somiglianze con il sistema sabellico e italico. Il fonema iniziale ${ }^{\star} b h$ - viene realizzato con la sorda corrispondente $/ \mathrm{b} /$ in ta-bara da ${ }^{\star} b h e r o-\left(c f\right.$. lat. fero, per ${ }^{\star} b h$ - in osco $c f$. far 'grano' da $\left.{ }^{\star} b h a r s\right)$. Il verbo hipades 'ha dedicato', ci offre un'evidenza del trattamento di ${ }^{\star}-d h$ $\left({ }^{\star}\right.$ supo- $\left.d^{h} \bar{e}-s-t\right)$, per il quale si offre in latino fumus $\left(<{ }^{\star} d h \bar{u} m o s\right)$ 'fumo', sudpiceno mefiín, mefiaí (< ${ }^{\star}$ medhyo-), osco rufriis, latino ruber (< ${ }^{*}$ reudh-) 'rosso'.

Per il trattamento delle labiovelari, l'unica evidenza è il nome personale Penkeos or Penkaheh[e?] ( ${ }^{*} p e n k^{w} e-{ }^{\prime}$ 'cinque'), lat. quinque; osco pomtis (<*pompe, 'cinque'; Untermann 2000, 603-604).

Un fenomeno di diffusa 'palatalizzazione', ovvero di scritture con doppia

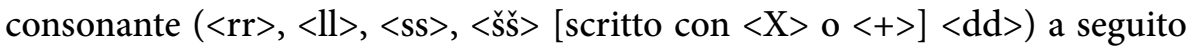
in incontro di consonante $+\mathrm{y}$, è stato studiato sin dagli esordi degli studi sul messapico, in primo da V. Pisani 1953, 247, poi da H. Krahe 1928, 81-104, da C. de Simone, da A.L. Prosdocimi e da V. Orioles 1972 e 1993. 
Recentemente J. Matzinger gli dedica un paragrafo del suo volume sulla lingua messapica $(2019,32-33)$ e A. Filippin c.s. ne accenna in un recente contributo ad un convegno sulle lingue sabelliche.

La documentazione viene qui riproposta in base alle occorrenze dei $M L M$, integrati con la documentazione apparsa dopo il 2002.

La palatalizzazione messapica è osservabile nei seguenti casi di incontro tra consonante e /y/:

- $\quad$ per $<$ ll $>$ si contano ad oggi 27 casi: ad es. bollihi, valla, vallaos, vallaos, poollo;

- $\quad$ per $<$ rr $>$ si contano 22 casi, tra cui haštorrihi, artorres, korrihi, fotorridas, $\Psi$ aotorrihi;

- $\quad$ per <nn> si hanno 21 casi: ad es. vallasso, dazohonnihi, šaillonna, dazinnihi, poššonnihi, ardannoa;

- la palatalizzazione di $/ \mathrm{t}+\mathrm{y} /$ assume la forma $\mathrm{di}\langle\mathrm{t} \theta>$, per la quale si

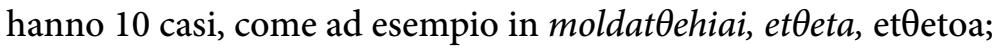

- $\quad$ per $<\mathrm{dd}>$ si hanno cinque casi, come ad es. dastidda, iddi;

- $\quad$ per <zz> cinque casi tra cui hazzava e mazzes;

- l'incontro di $\mathrm{s}+\mathrm{y}$ produce geminazione, come in $\theta$ aotarassi, arnisses, nekassihi, pessi;

- la spirante interdentale palatalizzata $\check{s}$, resa graficamente in messapico con il segno $\mathrm{X} \mathrm{o}+$, raddoppia all'incontro con /y/ in sette casi, come ad es. in vaššnihi, droššihi, oršson.

Per il PN maschile vǎšnihi, che presenta una consonante dopo la geminazione, l'analisi del prenome (su cui v. Untermann 1964, 176) deve essere fatta in diacronia. La forma presuppone una formazione in -na- su un tema in consonante: ${ }^{*} v a s ̌ s ̌-n a-$, la cui etimologia rimane oscura. Probabilmente il nome è da considerare imprestito e presentava la geminazione già al momento del suo ingresso nella lingua messapica.

Alcuni casi di palatalizzazione risultano poco attestati: $\langle\mathrm{s} \theta>$ in zaris $\theta e s$ : ${ }^{*} z a r$-istya-s $\left(<{ }^{*} z a r-i s t y o-s\right),<\mathrm{gg}>$ in epilaggi, di cui si ignora classe grammaticale ed etimologia $\left(\mathrm{da}^{\star}-g y\right.$-?), $<\mathrm{pp}>$ in kroppas $\left(\mathrm{da}^{\star} k r o p-y a-s\right.$ ?), per i quali non è esclusa la possibilità di imprestiti (ma da quale lingua?). Vanno infine ricordati due casi di $\langle\mathrm{hh}>$ : gelaihhihi e mahharaos per i quali il valore di /h/ come fricativo è solo un'ipotesi analogica con altri impieghi $\mathrm{di}<\mathrm{h}>$ in messapico. 
A partire da H. Krahe 1929 è stata avanzata una somiglianza tra la palatalizzazione messapica e quella di area osca. Raddoppiamento di consonanti all'incontro con /y/ si hanno infatti nelle forme osche velliam ( ${ }^{*}$ wel-ya; Rix $S T$ Cp 36), vítelliú ( ${ }^{*}$ witel-ya; Rix ST nPg 1b), akudunniad ( ${ }^{*}$ akudun-ya-d; Rix ST nSa 6), virriieís ( ${ }^{\star}$ wir-yo-; Rix ST Cp 24.27), úíttiuf ( ${ }^{\star}$ oityōns; Rix ST Cm 1B14.17), mamerttieís ( ${ }^{*}$ mamert-yo-; Rix $S T \mathrm{tCp} \mathrm{1),} \mathrm{\omega v \delta \delta ı \eta}{ }^{*}$ oud-j-Rix $S T$ Lu 24; cf. lat. Ouidius), dekkieis ( ${ }^{*}$ dek-yo-; Rix ST Cm 5), úppiis ( ${ }^{*} u p-y o-\propto$; Rix ST Cp 36), babbiis ( ${ }^{\star} b a b-y o-;$ Rix ST Hi 2) (cf. Orioles 1972, 71). Lanalogia di forme come quelle sopra elencate con quelle messapiche veniva spiegata dallo studioso tedesco con un influsso del Messapico nella zona osca, in particolare bantina. Pisani 1933 intravedeva nel fenomeno piuttosto l'esito di isoglosse tra lingue confinanti. Orioles 1972, 75, ricorda come comportamenti analoghi al bantino in fatto di palatalizzazione vadano piuttosto ricercati a nord, nei dialetti sabellici del Sabino, Peligno, Marsico e Umbro.

In realtà, se fenomeni di palatalizzazione e/o assibilazione interessano un cospicuo gruppo delle lingue sabelliche, le forme che questi assumono in messapico sono diverse da un punto di vista grafematico. Nella zona bantina ad esempio osserviamo piuttosto assibilazioni come zicolom ( ${ }^{\star}$ dyēkolom), meddixud ( ${ }^{*}$ meddikyōd), Bansae ( ${ }^{\star}$ bantyai), dovute all'incontro di consonanti + $/ y /$, anche se sporadicamente si osserva geminazione come ad esempio in allo ${ }^{*}$ aly $\left.\bar{a}\right)$. Le forme sopra elencate fuori da Bantia presentano sì raddoppiamento delle consonanti che precedono y, ma conservano l'elemento palatale y, scritto $<\mathrm{i}>$, che in Messapico si perde. In questo, se vogliamo, il Messapico dovrebbe essere messo a confronto con gli esiti di alcuni dialetti greci, dove ad es. in Eolico si osserva una palatalizzazione tramite raddoppiamento della consonante nell'incontro con la semivocale /y/ e la perdita di quest'ultima (Blümel 1982, 111). A differenza di questi però, anche la /s/ subisce in Messapico fenomeni di palatalizzazione, conservando forme raddoppiate come $\theta$ aotarassi (MLM 2 $\mathrm{Br}$, III sec.), arnisses (gentilizio maschile, nominativo; MLM $10 \mathrm{Cae}$, III sec.), nekassihi (gentilizio maschile, genitivo; MLM 16 Cae, III secolo). 


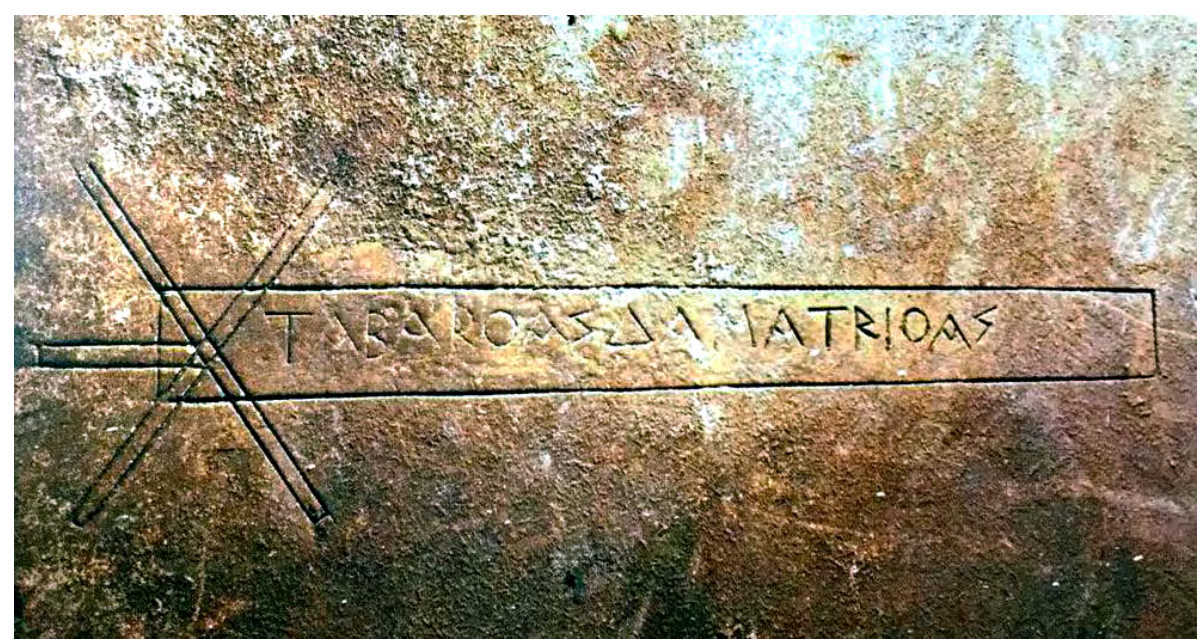

Fig. 2. Lastra di calcare pertinente ad una tomba a cassone da Valesio. MLM 16 Bal. IV sec. a.C.

L'iscrizione presenta un caso di nome sacro al genitivo, Tabara Damatrioa (ieronimia) iscritto entro una fiaccola demetriaca. (da Italia Omnium Terrarum Parens, Milano 1989).

Come esempi di restituzione di occlusiva $+/ \mathrm{y} /$ si possono citare il beotico

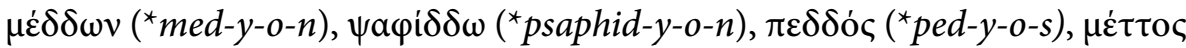

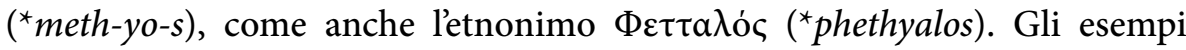
sopra citati ci spingono ad estendere la nostra comparazione al di fuori del mondo strettamente italico, dato che, come dicevamo sopra, non si verificano tra il mondo messapico e quello italico - in particolare osco, che è quello direttamente confinante con l'Apulia - quei fenomeni di osmosi e condivisione di elementi culturali, presupposto di ogni fenomeno di contatto linguistico.

Per quanto riguarda la cronologia delle attestazioni, su un totale di 620 iscrizioni, la maggior parte di grafie palatalizzate si hanno nel III sec. a.C. (70 casi), ovvero nella fase di maggiore evidenza documentaria. Almeno sette occorrenze si datano nel periodo tra VI e prima metà del V secolo, due sono nella seconda metà del V, otto tra IV e III secolo, e più tardi nove tra seconda metà III e fine II sec. a.C. Stando all'evidenza documentaria, il fenomeno della palatalizzazione in Messapico sembra un fatto già assestato in età pre-documentaria.

Da un punto di vista lessicale, si sono riscontrati fenomeni di palatalizzazione in gentilizi, prenomi (28/108), sia maschili (13) che femminili (12), forse due toponimi (vasmannati? e ardonnoa), teonimi (otto) e sei appellativi in funzione aggettivale. Del resto, possibili contatti di consonante e/-y-/ han- 
no avuto luogo nella lingua messapica in ogni occorrenza di formazione delle parole, laddove il suffisso $-y o /-y a-$ costituiva una modalità di derivazione dal tema di base, esattamente come avviene per le altre lingue italiche.

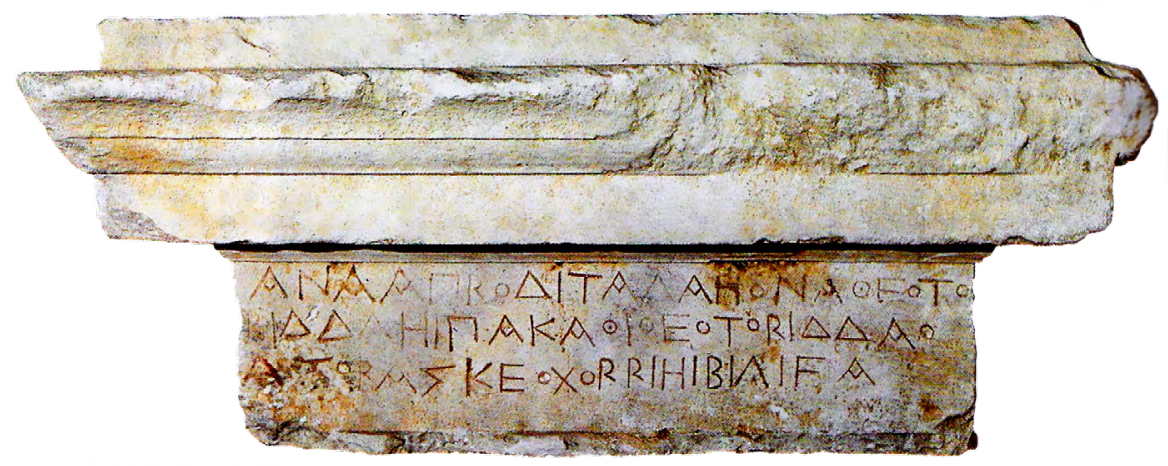

Fig. 3. Parte terminale di un pilastro di pietra leccese da Ceglie, loc. Monte Vicoli. MLM 22 Cae. III sec. a.C. L'iscrizione riporta una dedica ad Aprodita (da Italia Omnium Terrarum Parens, Libri Scheiwiller, Milano 1989).

In altre parole, il suffisso latino-italico ${ }^{*}-y o$ - comunemente usato come formante nei gentilizi è semplicemente un suffisso derivazionale, e si adatta sia ai nomi comuni sia ai gentilizi. In Latino -ius $\left(<^{*} y o-s\right)$ forma gentilizi come Iulius ( $<^{\star}$ Iul-yo-s) ma anche aggettivi derivazionali patrius (Leumann 1977, 263 ; cf. Rix 1972, 718-720).

\subsubsection{Morfologia}

La morfologia del Messapico presenta un alto livello di frammentarietà, dovuto non solo al basso numero di documenti pervenuti fino a noi, quanto anche alla tipologia dei testi conservati, per lo più di ambito funerario. Sicuramente sono attestati due numeri: singolare e plurale. Per il genere, non c'è evidenza del neutro, che è comunque difficile da individuare: l'adattamento di un imprestito greco neutro, à pyúpıov 'argento', trattato come argorian, non ci fa capire se la vocale tematica sia effettivamente un femminile dei temi in $-a$ o la resa di $/{ }^{*} \breve{c} /$. Si ha evidenza di quattro casi: nominativo, accusativo, genitivo e dativo. In un caso sembra attestato un locativo, ovvero nel toponimo Vasmannati (MLM $1 \mathrm{Br})$. 
Sono state individuate le seguenti classi tematiche:

\begin{tabular}{|c|c|c|}
\hline $\begin{array}{c}\text { Temi in }-\breve{a} \\
\text { (variante }-o a)\end{array}$ & Singolare & Plurale \\
\hline Nom. & $-a($ es. An $\theta e t a)$ & - \\
\hline Gen. & -as (Baoštas) & - \\
\hline Dat. & $-a($ Ana Aprodita $)$ & -abis (Vale $\theta a b i s)$ \\
\hline Acc. & -an (Aproditan) & \\
\hline
\end{tabular}

\begin{tabular}{|c|c|c|}
\hline Temi in $-y \breve{a}$ & Singolare & Plurale \\
\hline Nom. & $-i a$ (bilia 'filia', 'figlia') & - \\
\hline Gen. & -ias (bilias) & - \\
\hline Dat. & - iai (Arzeriai) & - \\
\hline Acc. & -ian (]tarpalian?) & - \\
\hline
\end{tabular}

\begin{tabular}{|c|c|c|}
\hline Temi in $-i$ & Singolare & Plurale \\
\hline Nom. & -is (Aiddetis) & - \\
\hline Gen. & -eos (AviӨeos) & - \\
\hline Dat. & $-i($ Vareti $)$ & -ibas (Logetibas) \\
\hline Acc. & -in (danin?) & - \\
\hline Loc.? & $-i ?$ (Vasmannati, topon.) & - \\
\hline
\end{tabular}

\begin{tabular}{|c|c|c|}
\hline Temi in $-\boldsymbol{u}$ & Singolare & Plurale \\
\hline Nom. & -os(Etos, Morkos) & - \\
\hline Gen. & -oaihi (VasOoaihi) & - \\
\hline Dat. & & - \\
\hline Acc. & - & - \\
\hline Loc. & & - \\
\hline
\end{tabular}

\begin{tabular}{|c|c|c|}
\hline Temi in $-\boldsymbol{n}$ & Singolare & Plurale \\
\hline Nom. & $\begin{array}{c}\text { [+palat] }(\text { i)o (Vallasso, Akello, } \\
\text { Ošš, Gaorio })\end{array}$ & - \\
\hline Gen. & $\begin{array}{c}\text { [+palat.]-onas (Molonas, } \\
\text { KriӨonas, Dazihonas, Baledonas })\end{array}$ & - \\
\hline Dat. & - & - \\
\hline Acc. & - & - \\
\hline Loc.? & - & - \\
\hline
\end{tabular}




\begin{tabular}{|c|c|c|}
\hline $\begin{array}{c}\text { Temi in }-\boldsymbol{r} \\
(- \text { tōr })\end{array}$ & Singolare & Plurale \\
\hline Nom. & -tor $(\Theta a$ aotor $)$ & - \\
\hline Gen. & -toras $($ (aotoras, kalatoras $)$ & - \\
\hline Dat. & -tori $($ Taotori $)$ & - \\
\hline Acc. & - & - \\
\hline Loc? & - & - \\
\hline
\end{tabular}

\begin{tabular}{|c|c|c|}
\hline Temi in $-\boldsymbol{t}$ & Singolare & Plurale \\
\hline Nom. & $-t$ (Bosat, Dazet $)$ & -tes (Brinnaštes $)$ \\
\hline Gen. & - tas $($ Daštas, Plastas $)$ & - \\
\hline Dat. & - tei (Dazetei $)$ & - \\
\hline Acc. & - & - \\
\hline Loc.? & - & - \\
\hline
\end{tabular}

\begin{tabular}{|c|c|c|}
\hline Temi in $-\boldsymbol{s}$ & Singolare & Plurale \\
\hline Nom. & - as (Venas) & - \\
\hline Gen. & - & - \\
\hline Dat. & - & - \\
\hline Acc. & - & - \\
\hline Loc.? & - & - \\
\hline
\end{tabular}

Tab. 2. Classi tematiche nominali.

Un problema particolare della morfologia messapica è rappresentato dal genitivo singolare dei temi in - $a$ e $-y a\left(\mathrm{da}^{*} o \mathrm{e}^{\star} y o-\right)$, ovvero - $a i h i \mathrm{e}-i h i$, morfemi tra i più rappresentati nella documentazione epigrafica, dato che nelle iscrizioni funerarie la formula testuale è l'espressione del nome personale (in nominativo o in genitivo) seguito dal gentilizio o patronimico, formati proprio dal suffisso derivazionale $-y o-$ e dal genitivo (-ihi). Il tema investe non solo il Messapico, ma anche la documentazione di altre lingue i.e. occidentali, che presentano forme diverse di genitivo singolare e che vengono spesso richiamate a confronto nella letteratura specialistica. Da una parte infatti, considerando il grafema $<\mathrm{h}>$ messapico un espediente grafico per allungare la vocale successiva, si tende a considerare la forma -ihi come equivalente a - $\bar{l}$ (De Simone 1992 e da ultimo 2017, 1845). In altri casi si considera che il grafema $<\mathrm{h}>$ abbia valore fricativo, e che quindi la forma -ihi costituisca un'e- 
voluzione del genitivo -osyo, documentato anche nel Latino arcaico (si veda ad esempio il popliosio valesiosio del Lapis Satricanus, fine VI sec. a.C.), in celtico (Castelletto Ticino: xosioiso e plioiso di Castelletto Ticino, Rondineto e S. Fermo, VI e V sec. a.C.) e in venetico (kaialoiso di Oderzo, V sec. a.C.). Quest'ultima opinione è stata sostenuta già da V. Pisani (per il Latino-Falisco, 1964, 344-345; 1971, 181; 1974, 149-151, poi da R. Gusmani 1976a; 1976b; 2006, da V. Orioles 1981; 1989 e da A.L. Prosdocimi 1989, 137-174; 1990; 2006.

Un'obiezione sollevata alla soluzione $-i h i=-\bar{i}$ è che negli altri casi di conservazione del suffisso (in Latino e in Celtico ad esempio), la vocale tematica non è conservata, confluendo in $-\bar{\imath}$, mentre in messapico rimane sempre distinta. D'altro canto, la soluzione che -ihi derivi da -osio implica una trafila ricostruttiva che pecca di lacune documentarie. Se -osio è infatti attestato nella documentazione epigrafica, lo sviluppo intermedio -oyyo (secondo Pisani 1971,181 ), che spieghi il passaggio da -osio a $-i$ non lo è (Lejeune 1989, 74). Per un uso diacronico da -osyo a - $i$ sembrerebbe parlare anche l'evidenza falisca, nella quale le attestazioni dei due morfemi si distribuiscono così: le forme *euotenosio, kaisiosio, aịmiosio sono nell' "Early Faliscan", mentre i genitivi in - $i$ appaiono nella documentazione del "Middle" e "Late Faliscan" come uipi, caui, cesi, marci (Bakkum 2009, 129-130). La stessa distribuzione complementare tra età arcaica e recente per i due suffissi era già stata notata da Lejeune per il Venetico (ma una sola attestazione di -oiso!) Latino e Leponzio (Lejeune 1989, 74-77; cf. Ciceri 2012, 73-74).

Se analizziamo il valore del grafema $<\mathrm{h}>$ in tutta la documentazione messapica, troviamo che esso è attestato finora 58 volte, di cui otto inutilizzabili per incompletezza dell'iscrizione. In 18 casi il grafema è impiegato in casi di palatalizzazione dovuta all'incontro di consonante $+-y$-. Tre volte (hipades, hadive e forse hamipallen), la $h$ - ̀̀ stata ricondotta a $s$ - ereditata. In due casi si ha una successione di due $<\mathrm{h}>$, ma la parola è incompleta. Tutti gli altri casi sono probabili $h$-originari (tra questi hazzan/hezzan con i derivati hazzava, hazzavoa, hazavidihi, hazava, oppure haivahias, scritto anche haivaYias. Quest'ultimo caso appare significativo, perché il grafema $\Psi$ appare come soluzione arcaica al posto $\mathrm{di}<\mathrm{h}\rangle$. Si suppone che questo segno a tridente, presente anche in bale $Y_{\text {ias }}(M L M 15 \mathrm{Bal}$ ), possa avere avuto una valenza fricativa molto vicina a $/ \mathrm{h} /$ e che quindi in seguito sia stato da esso sostituito ( $c f$. sopra, $\$$ 1.4.1). Appare chiaro, a mio avviso, che il grafema $<\mathrm{h}>$ può aver coperto una serie ristretta ma differenziata di valori fonologici, come succede del resto per il grafema $/ \theta /$, e che possa aver rappresentato una fricazione derivata da 
$-y$ - nei casi di genitivo, come anche un'aspirazione in caso di sviluppo $\mathrm{di}^{\star} s-$. Il Messapico potrebbe aver rappresentato, nelle forme di genitivo singolare dei temi in - $a$ e $-y a$, proprio il suono rimasto dallo sviluppo di -sy-, scritto in età arcaica con i grafemi $\Psi, \Psi, \uparrow$. Non ci sarebbe stato bisogno infatti di creare dei nuovi grafemi se $<\mathrm{h}>$ avesse da solo coperto tutti i valori. In diacronia probabilmente i suoni si sono unificati, tanto da essere rappresentati dal solo $<\mathrm{h}>$, ma quello che ci interessa è proprio l'inizio della documentazione, che riproduce un valore originario differenziato dei grafemi.

Quanto detto non risolve però il rapporto tra le due forme ${ }^{*}-i$ e -o-syo-. La coincidenza di $-\bar{\imath}\left(<\mathrm{da}-\varnothing \bar{\imath}<{ }^{\star}-y H\right)$ con il suffisso di 'mozione' femminile i.e. ${ }^{\star} \bar{i}$, o meglio la sua derivazione da questo nel valore di "appartenenza" (su cui Tichy 1993, $c f$. Marchesini 2013b per i rapporti tra mozione italica ed etrusca), può motivare la separazione dei due suffissi sin dall'inizio. In quest'ottica - $i$ ed -osyo possono essere due cose distinte, venute sempre più a coincidere formalmente in età storica.

\subsection{I testi: numero di iscrizioni, generi epigrafici, tipologia dei supporti.}

Dopo le 545 iscrizioni messapiche pubblicate nel 2002 (MLM) cui si debbono aggiungere 48 testi considerati dubbi per tratti epigrafici non caratterizzanti, sono state pubblicati, come già ricordato sopra, pochi altri testi $(\$ 1.3)$. Come si può vedere sulla fig. 4 , la maggior parte di questi viene da Alezio (53 testi), che presenta un'epigrafia sostanzialmente tombale. All'interno delle tombe a cassone in pietra locale (càrparo), sono iscritti il nome e il gentilizio della persona sepolta all'interno (per un quadro sull'onomastica di Alezio cf. de Simone 1982). Segue Ostuni, che dopo gli ultimi ritrovamenti conta un totale di 53 testi, su ceramica da tavola e su ceramica grezza. Da Lupiae (Lecce) provengono 50 iscrizioni, quasi tutte dalla necropoli dell'Anfiteatro. Molte di queste sono sigle o lettere singole, in parte abbreviazioni onomastiche. Altre poche iscrizioni provengono dall'area cittadina, ma comunque da tombe singole, e poche altre (tre) provengono dal territorio intorno alla città. Segue Mesagne (Brindisi), con 41 testi provenienti per lo più dalla Contrada Muro Tenente, e da contesti funerari. Anche Ceglie Messapica, Gnathia, Rudiae, Vaste, Valesio, Oria, Ruvo e Vereto hanno restituito una trentina di testi. Per il resto, le iscrizioni decrescono numericamente, e in molti centri se ne sono rinvenute in poche unità.

È significativo che le iscrizioni più antiche provengano da quelle città come Oria, Mesagne, Muro Tenente a Valesio - che si dotano durante il VI 
secolo di unarchitettura tombale, iniziando a seppellire i defunti entro cassoni di pietra (Lombardo 1994). Come detto sopra, le zone settentrionali, in Peucezia e Daunia, presentano un'epigrafia tarda, e limitata.

La Daunia, con Arpi, Canosa, Salapia e Vieste Garganico, ha restituito 28 iscrizioni, la maggior parte su pesi da telaio. La Peucezia, con i centri di Adelfia, Gravina, Laterza, Monopoli e Ruvo, ha restituito nel complesso 28 testi, in gran parte dall'ultimo centro, e anche in questo caso quasi tutti su pesi da telaio. Per questo ambito settentrionale sono state avanzate delle ipotesi rispetto alla cultura scritta come in parte 'sostituita' dalla cultura visiva dei grandi vasi apuli decorati con scene del mito e del teatro greco (vedi supra, $\S$ 1; Todisco 2011; 2013; Marchesini 2013a; c.s. 3).

Ci si domanda infatti come l'assenza o comunque la scarsa evidenza epigrafica epicoria si possa combinare con unacculturazione rispetto anche alla cultura e al mito greco assai evoluta, come riflesso nel repertorio figurativo espresso nei grandi vasi apuli. Rispetto al mondo messapico, che nutre rapporti spesso conflittuali rispetto a Taranto, il nord della Apulia sembra impostare il suo programma culturale avvalendosi proprio del mito e del teatro greco, utilizzato per la celebrazione delle élites locali, per rivendicare una propria autonomia culturale. $\grave{E}$ da intendere in questa direzione probabilmente anche la scelta alfabetica di adeguare la tipologia delle lettere più a modello greco che a quello messapico, reintroducendo ad esempio il grafema $\langle\mathrm{Y}\rangle$, che l'epigrafia messapica aveva abolito (su questo aspetto in particolare $c f$. Marchesini 2013a).

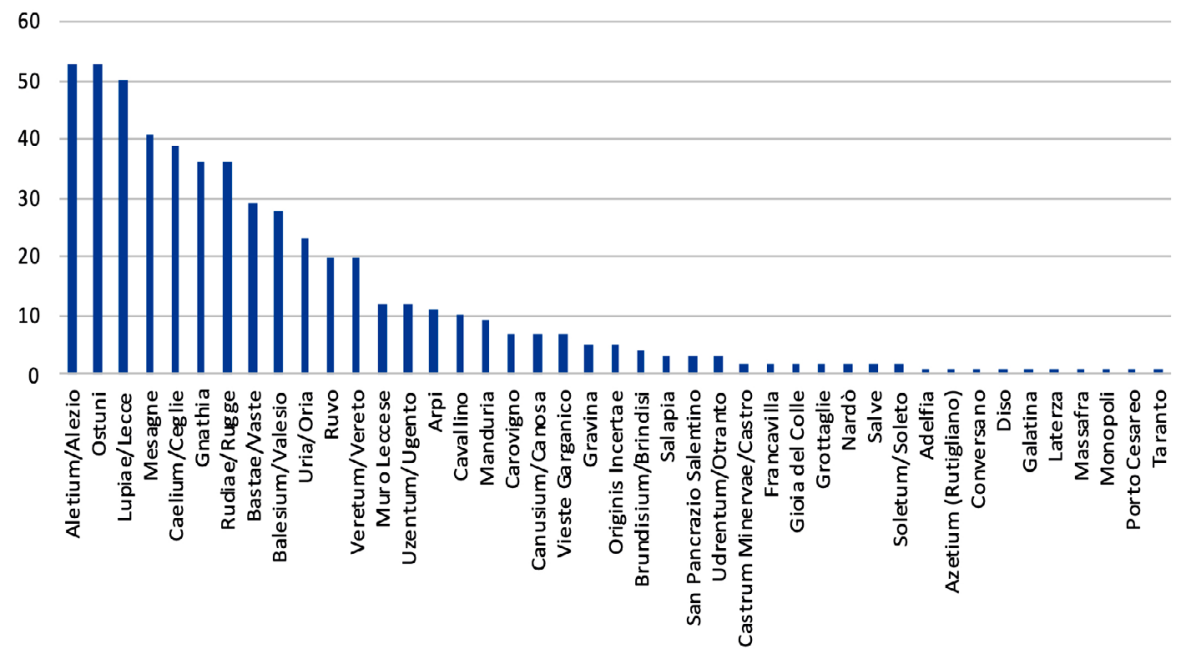

Fig. 4. Provenienza delle iscrizioni messapiche. 
Un'altra considerazione deve essere fatta in relazione alla tipologia di supporti epigrafici (fig. 5), che condizionano anche la tipologia testuale. Come detto sopra, se facciamo astrazione dal complesso epigrafico di Grotta Poesia, con un numero imprecisabile di iscrizioni, la classe più rappresentata di supporti epigrafici è quella delle tombe, con un totale di 209 unità. Segue la ceramica fine, con un alto numero di vasellame proveniente da Ostuni, con 87 unità. Tra questi vi sono anche vasi con alto valore artistico, come le sette trozzelle da Oria, Valesio e Rudiae, che presentano nomi personali per lo più dipinti (MLM 21 e 35 Ur, 2 e 19 Bal, 14, 15 e 21 Rud).

Anche i pesi da telaio sono ben rappresentati (59 casi), soprattutto al nord: la maggior parte viene da Arpi e Ruvo, e presenta nomi personali, sia maschili (Platur, Vinnadeus) che femminili (Artita, @eniana, Epaka, Dazia). Per alcuni nomi, espressi in nominativo, e per le sigle di due o tre lettere, si è proposta una identificazione come 'trade mark' (Marchesini 1995). Per i cippi e le colonnette iscritte, in numero di 26, Mario Lombardo ha evidenziato come essi accompagnino la monumentalizzazione delle tombe (vedi sopra) avvenuta tra VI e V secolo a.C. e quindi abbiano piuttosto una funzione funeraria che civica o urbana (Lombardo 2013).

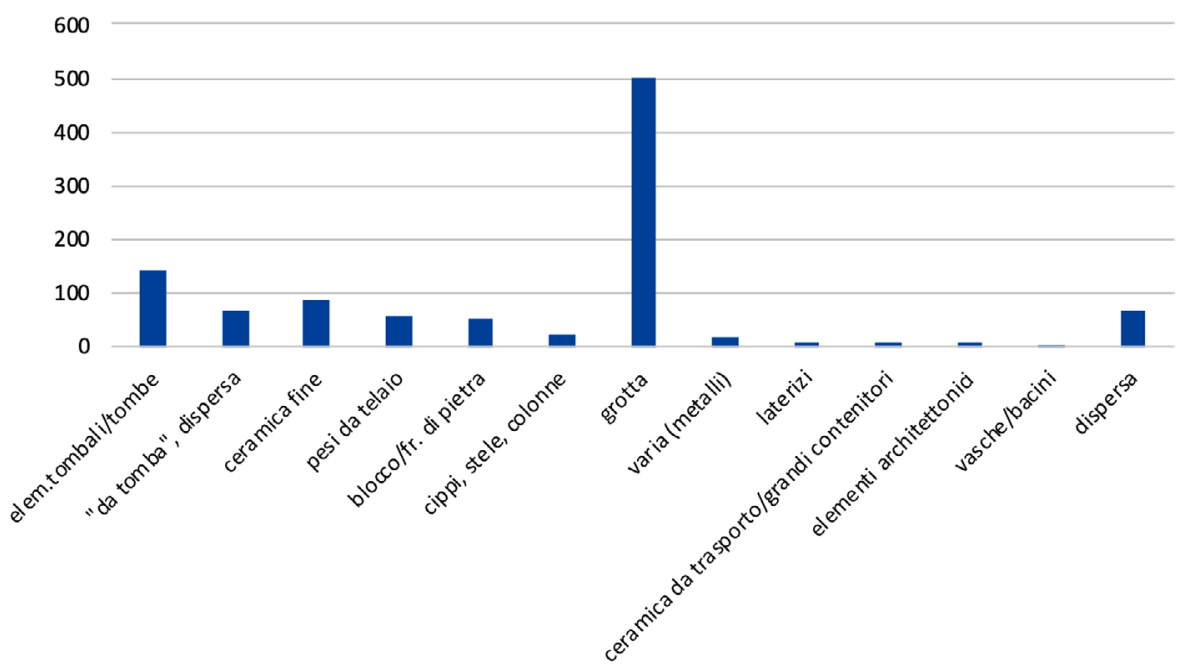

Fig. 5. Tipologia dei supporti epigrafici. 
Ad architettura civile, e quindi con fruizione pubblica, si riconducono gli elementi architettonici (otto) o altri blocchi e lastre di pietra (55), che spesso contengono iscrizioni più lunghe, talvolta introdotte dall'invocazione a Zeus. Tra queste vale la pena di citare l'iscrizione lacunosa, di ca. 15 parole, da Oria MLM 22 Ur (III sec. a.C.), dalla grotta di Monte Papalucio, o l'iscrizione su blocco di càrparo da Francavilla MLM $1 \mathrm{Fr}$ (del III sec), con una decina di parole, anche questa lacunosa. L'iscrizione più lunga è senz’altro quella su blocco o lastra di pietra da Brindisi, purtroppo nota solo da un apografo di De Leo (da cui prende nome la biblioteca arcivescovile brindisina in cui era conservato il documento) copiato da Mommsen 1848, con ca. 70 parole. Questo documento è importante anche per aver conservato, soprattutto nel testo b), meno lacunoso di a), non solo l'invocazione a Zis all'inizio del testo (klaohi $Z i s$, "ascolta Zis"), ma anche alcuni lemmi di notevole importanza: il nome vasmannati, un possibile toponimo per il quale è stato proposta una forma di dativo/locativo (MLM II, s. v.), il termine argorian, presente anche in un'iscrizione da Salve (MLM $1 \mathrm{~Sv}$ ), noto anche nel composto argorapandes in MLM 1 Car, probabile imprestito dal greco ả pyúpıov ('argento', 'denaro'?). L'espressione ma berain, con la particella proibitiva ma seguita dal verbo 'portare' $\left({ }^{\star} b^{h}\right.$ eroint, ottativo da ${ }^{\star} b^{h}$ er- lat. fero). Purtroppo, il contenuto dell'iscrizione, nonostante la sua lunghezza, rimane per noi inaccessibile, anche perché non sono noti dati contestuali sul ritrovamento della lastra.

Per quanto riguarda la tipologia dei supporti epigrafici, considerando i dati dei $M L M$ aggiornati con i successivi ritrovamenti, il numero senzaltro più elevato di iscrizioni è praticata su pietra (302 unità). Di questi la maggior parte è da riferire a lastre di càrparo (pietra calcarenitica locale) pertinenti a tombe (209 unità). Le iscrizioni sono qui generalmente incise allinterno della cassa, come ben mostrano le iscrizioni di Alezio. Ad iscrizioni su pietra appartengono anche 55 blocchi di pietra, integri o frammentari, 27 tra cippi, stele e colonne, otto elementi architettonici e tre bacini di pietra. Il numero più elevato di iscrizioni su pietra è quello delle iscrizioni incise sulle pareti rocciose di Grotta Poesia, che ammontano a diverse centinaia. 


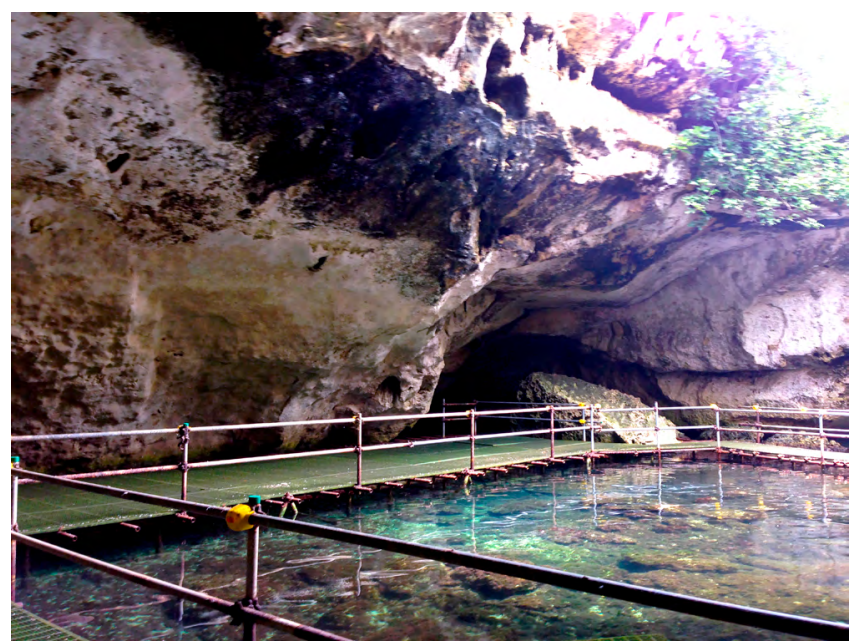

Figs. 6a-b. Grotta Poesia, Roca (Melendugno), veduta generale (a) e parete iscritta (b). IV-II sec. a.C. (foto dell'autore, consenso alla pubblicazione della Soprintendenza Archeologia Belle Arti e Paesaggio per le Province di Brindisi, Lecce e Taranto).

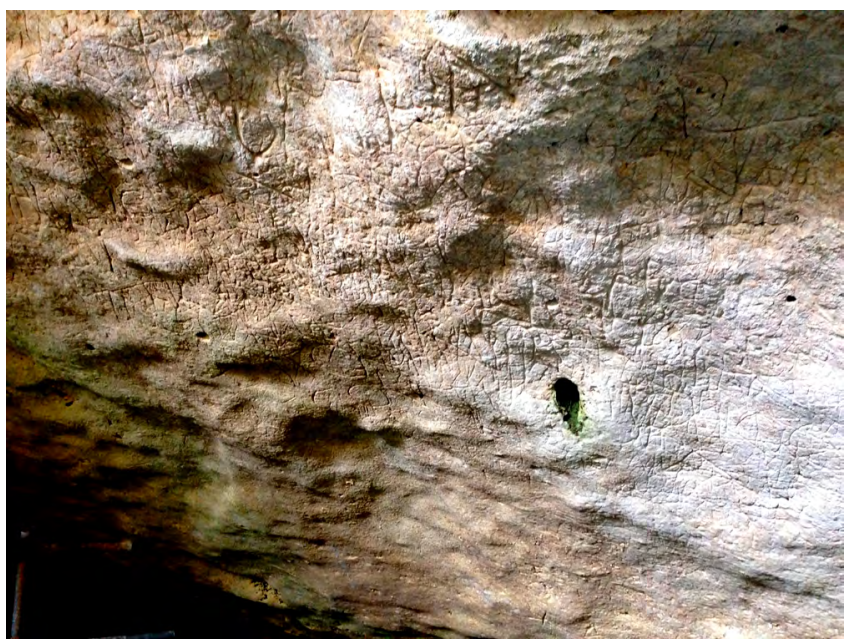

Su ceramica si registrano 165 iscrizioni, di cui 87 su coppe di ceramica da tavola, 59 pesi da telaio, assai diffusi soprattutto in Daunia, nove tra tegole e coppi e 10 su grandi vasi o anfore da trasporto. Anche il metallo è utilizzato, anche se non in modo esteso: su 18 oggetti in metallo recanti iscrizioni, abbiamo un disco di bronzo, 12 lastre di bronzo, un caduceo di bronzo, un astragalo di bronzo con iscrizione 'magica', una teca di argento con iscrizione realizzata con tecnica di puntinatura. 
Per quanto riguarda le monete, manca ad oggi un corpus completo e onnicomprensivo, ma diversi lavori di sintesi sono stati proposti da A. Stazio prima $(1971 ; 1972)$ e da A. Siciliano poi $(1993 ; 2001)$. Le città di Arpi, Ausculum, Azetium, Barium, Butuntum, Caelia, Canusium, Grumum, Hyrium, Luceria, Neapolis, Salapia, Silvium, Teanum e Venusia (in Apulia), Baletium, Brundisium, Graxa, Orra, Neretum, Sturnium e Uxentum emettevano moneta in area propriamente messapica (Salento). Tra le emissioni apule, solo Baletium coniò monete di argento, mentre Arpi, Caelia, Canusium, Ruvi, Teate e Neretum coniarono sia in argento che in bronzo. Le altre città coniarono solo in bronzo.

Le monete apule, come spesso accade nella monetazione italiota e siceliota, coniarono usando caratteri greci, solo Teanum ha leggenda osca e poi latina. Baletium, Orra e Uxentum presentano legende in caratteri messapici. In genere il numero di monete per ogni emissione è scarso e ha breve durata. $\mathrm{Al}$ cuni centri di emissione non sono inoltre stati identificati: tra questi Grumum (Grumo Appula?), Sturnium e Graxa (cf. Ruotolo 2010, 19-22). Il periodo di emissione si inquadra tra la fine del IV e il III secolo a.C.

La cronologia delle iscrizioni messapiche è stata rivista in occasione della redazione dei $M L M$, che ha consentito di processare tutti i testi e di stabilire un limite superiore della prima metà del VI sec. a.C. e uno inferiore della fine del II sec. a.C. Il processo di romanizzazione, che si conclude nel 45 a.C. con la lex iulia Municipalis, inizia già nel corso III secolo a.C. che rimane comunque il secolo di maggior produzione epigrafica. Il fenomeno è ben evidente nella documentazione epigrafica di Grotta Poesia, dove le iscrizioni latine si sovrappongono, in numero molto ridotto a quelle messapiche.

\subsection{La scrittura}

Lo sviluppo epigrafico messapico vede distribuirsi nell'arco di quattro secoli ben sette fasi epigrafiche, di cui due considerate di transizione (fig. 7).

Come si vede dal grafico, la produzione epigrafica è costante nei secoli, iniziando con 50 iscrizioni nella prima fase, 50 nella seconda, con un incremento nella terza e quarta fase nel corso del IV secolo fino alla prima metà del III, per poi avere un consistente incremento nella seconda metà del III secolo, l'età del massimo sviluppo epigrafico. Come si vede, nell'ultima fase, la VII, che copre dalla fine del III secolo alla fine del II, sono note solo 33 iscrizioni. 


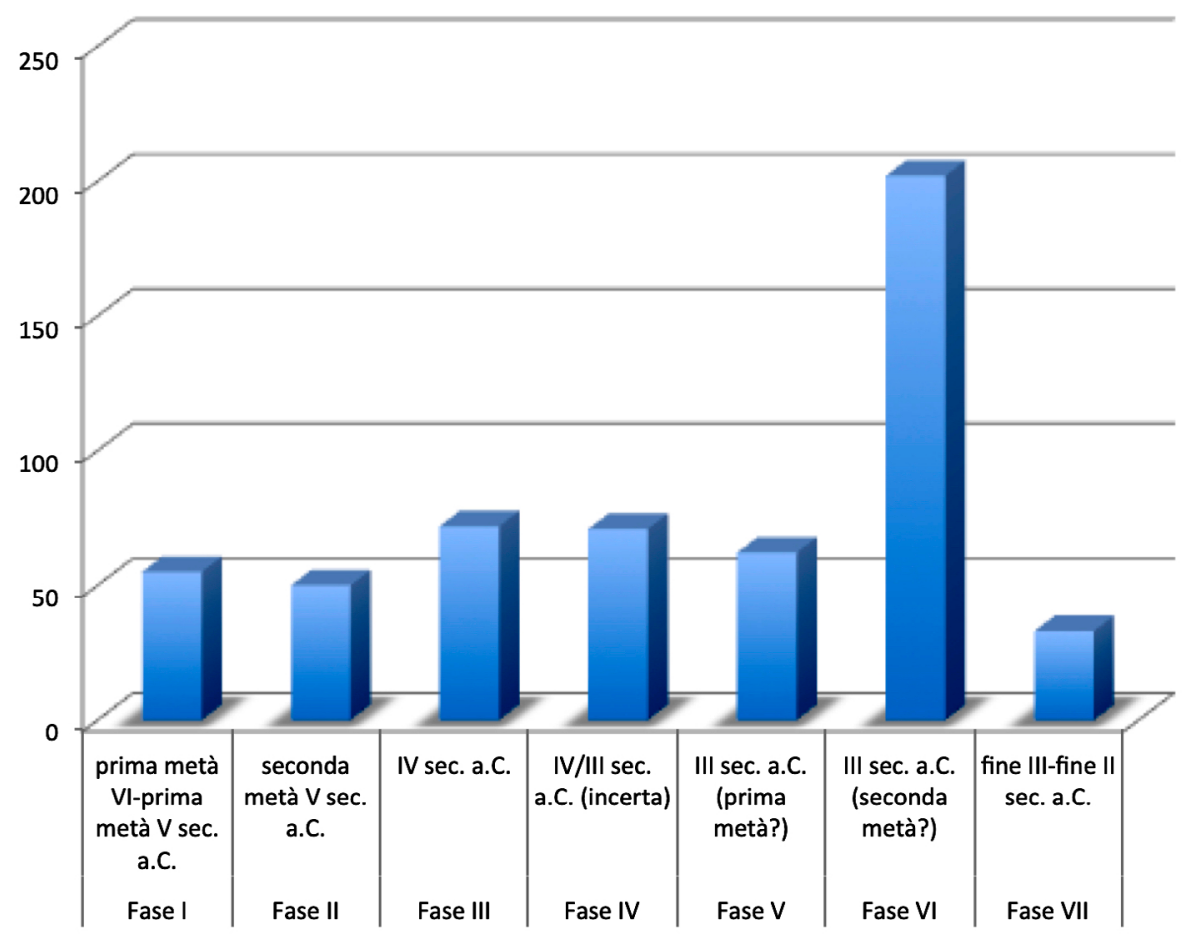

Fig. 7. Fasi epigrafiche: dati quantitativi.

Per quanto riguarda il ductus, le iscrizioni messapiche sono solitamente scritte da sinistra a destra, con alcuni casi più antichi di boustrophedon o di falso boustrophedon. La grafia è generalmente normalizzata, eccetto che nel periodo più antico, e l'esecuzione delle lettere tende sempre più verso uno stile con le 'grazie' (serifs), con segmenti delle lettere arrotondati e tratti brevi angolati o arrotondati ( $c f$. fig. 8).

Da un'analisi degli errori di scrittura (Marchesini 2004) è emerso che il Messapico ne presenta pochi, segno di una 'authority of writing' presente dall'inizio alla fine della produzione epigrafica. Il concetto di una autorità delegata al controllo delle attività di scrittura è introdotto da Coulmas 2003, 225, che riassume in questo termine quattro diversi aspetti funzionali della scrittura: la scuola, la coltivazione della lingua scritta ("perfect words"), la sua standardizzazione e la contabilità. L'assenza di errori, come anche lo sviluppo nei secoli di una tecnica scrittoria "calligrafica" che fa uso di grazie, sono alcuni dei segni tangibili della presenza di un controllo normativo sulle attività di scrittura. 


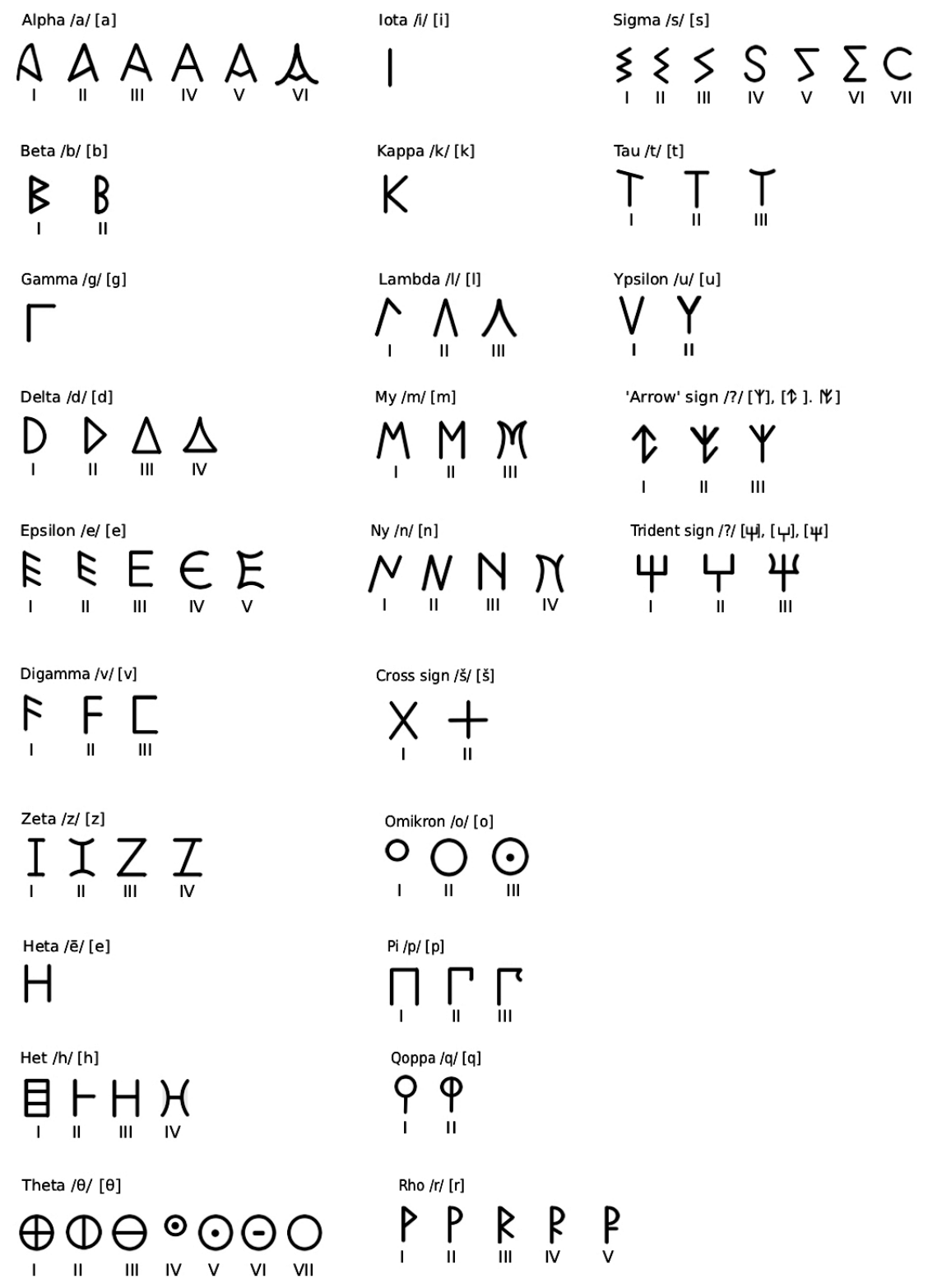

Fig. 8. Alfabeto messapico. I tipi sono orientati cronologicamente (grafica: S. Marchesini). 


\subsection{L'onomastica}

I Messapi, come il resto delle popolazioni dell'Italia preromana, hanno avuto un sistema di designazione basato sulla formula binomia, costituita da prenome e gentilizio. Bisogna però distinguere tra formule maschili e formule femminili, che subiscono modifiche in diacronia.

Il sistema onomastico messapico fu oggetto di studio di J. Untermann, che nel 1964 presentò uno studio sincronico e diacronico dellevidenza documentaria. Come rileva C. de Simone 2013, 53, l'impostazione di Untermann rimane sostanzialmente valida ancor oggi, e nessuna nuova evidenza ha sostanzialmente modificato le sue considerazioni (si vedano anche de Simone 1972, 192-201; Däuber 1991; de Simone 1982).

È merito di J. Untermann aver individuato nel secondo nome delle formule onomastiche - soprattutto le maschili - un gentilizio e non un patronimico. In questo senso la società messapica partecipa all'usanza etrusco-italica di designare la persona con un prenome seguito da un nome ereditabile, fatto che ad esempio non succede in Grecia, dove il nome aggiunto è solitamente di tipo patronimico o etnonimico. Questo dato è rilevante di per sé, dato che all'interno del mondo indoeuropeo le lingue che nel corso del I millennio presentano questo tratto si trovano solo in Italia, come già aveva messo in evidenza Rix 1972. Se escludiamo le lingue della Sicilia preromana, la cui scarsa documentazione impedisce una determinazione sicura, solo il Venetico presenta una soluzione 'eccentrica', non presentando evidenza di sistema gentilizio, se non sporadicamente (Untermann 1961).

Merito di Untermann è anche aver rilevato una differenza tra formule maschili e femminili. Le maschili sono tutte bimembri, costituite da prenome e gentilizio concordati tra loro in genitivo o nominativo. In alcuni casi si ha un solo prenome o un solo gentilizio, usato in modo assoluto. Per questi casi bisogna considerare che le condizioni pragmatiche di impiego (la situazione specifica, il contesto) possono aver reso ridondante l'uso di entrambi i nomi.

In pochi casi, già individuati da Untermann, la formula è trimembre, con l'espressione della filiazione (nome del padre in genitivo) dopo i primi due elementi della formula. 
Fig. 9. Cippo da Otranto, via del Porto, cantiere 2, dalla zona presso la porta urbica dell'insediamento messapico (in giacitura non originaria)

MLM 2 Hy. III sec. a.C. L'iscrizione riporta la formula onomastica maschile bimembre Daštas Taotorrihi. (Foto: Dpt. Beni Culturali, Università del Salento, consenso alla pubblicazione della Soprintendenza Archeologia Belle Arti e Paesaggio per le Province di Brindisi, Lecce e Taranto).

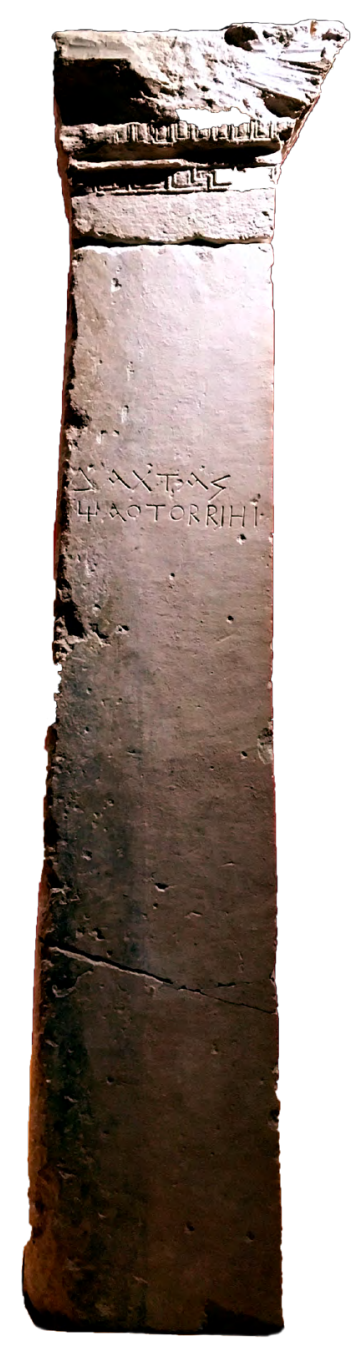

Per quanto riguarda i nomi femminili, si osservano casi di formule monomembri e bimembri, con prenome e appositivo. Per questo secondo caso si danno varie possibilità, ampliate rispetto a quanto osservato da Untermann, come messo in rilievo Däuber 1991, 326:

- $\quad$ Prenome femminile + appositivo femminile (gentilizio o patronimico; es. Dašta MorӨana, MLM 11 Cae, III sec. a.C.);

- $\quad$ Prenome femminile + patronimico + filiazione (nome del padre in

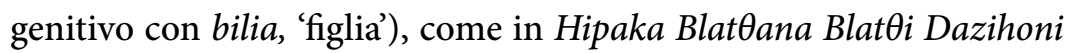
bilia (MLM 19 Ur, III sec. a.C.); 
- Prenome femminile + nome maschile (mono o bimembre in genitivo), come EtӨetoa Siillonas (MLM 2 Rud, del III sec. a.C.);

- Appositivo femminile + nome maschile in genitivo (senza prenome), come in Šaillonna Lomiaihi (MLM $25 \mathrm{Al}$, VI-prima metà V sec. a.C.).

- Formula trimembre con prenome, appositivo e gentilizio del marito in genitivo, come emerso da un'iscrizione di Grotta Poesia, dazoma

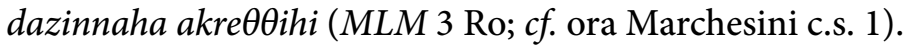

Diversi sono i suffissi che caratterizzano il nome aggiunto, sia esso il gentilizio dei maschili o il patronimico dei femminili: oltre a $-y a\left(<{ }^{*}-y o\right)$, si registrano anche i suffissi femminile -ana (es. BlatOana, Divana), il maschile -ides (< ${ }^{*}$ idyos) (es. Vallaidihi, Porvaides). Per -ya e -ides de Simone (2013, 56) osserva che potrebbero essere di comune eredità indoeuropea, piuttosto che di imprestito dal mondo italico, dato che mancano effettivi fenomeni di scambio onomastico tra i due ambiti linguistico-culturali.

\section{Problemi nello studio del Messapico}

Oltre alle difficoltà di acquisizione del complesso epigrafico di Grotta Poesia, cui accennavamo sopra, la maggior crux sulla lingua messapica sorge dal suo isolamento linguistico. I tentativi di una sua attribuzione linguistica ad ambito balcanico o di un confronto diretto in particolare con l'Albanese (Krahe 1955; Matzinger 2009, 2015, da ultimo si veda però Matzinger 2019), si scontrano con due ordini di difficoltà. Ciò che chiamiamo 'Illirico' è l'esito di una determinazione geografica delle fonti antiche, che indicavano con ciò una regione della costa orientale del Mare Adriatico, compresa tra i fiumi Arsa e Drin (Plin. Nat. 36) i cui abitanti vengono designati come Illyri proprie dicti (Nat.22.144). Le sole testimonianze epigrafiche provenienti da questa regione sono relative a nomi personali scritti in alfabeto greco o più tardi latino, di etimologia spesso non-greca (noti sono ad esempio $A \beta \alpha, A \beta \alpha \iota{ }^{\prime}$; $B \rho \iota \kappa \varepsilon v(v) \alpha$;

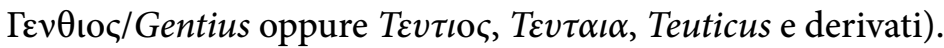

La seconda difficoltà è il confronto con una lingua, come l'Albanese, conosciuta da fonti scritte che iniziano con il XVI sec. d.C. e che rappresenta quindi un ulteriore livello di sviluppo linguistico e una maggiore distanza dalla protolingua indo-europea. Un confronto che possa 'reggere' tra due lingue o sistemi linguistici si deve presentare come una costellazione sistemica di tratti comuni, dalla fonologia, alla morfologia, alla sintassi. I tratti lessicali, spesso richiamati per il confronto tra lingue, sono i primi a 'saltare' quando le lingue si separano (Meillet 1925, 13; Mayrhofer 1986, 14; più recentemente 
Nichols 1996, 44) e quindi presentano un minor grado di attendibilità per la ricostruzione. Per un confronto tra Messapico e Albanese non si ravvisa un quadro sufficiente di tali corrispondenze, considerato soprattutto che il repertorio lessicale spesso chiamato a confronto si presenta come un tratto poco affidabile nella comparazione, a causa della grande distanza documentaria tra le due lingue.

\section{Riconoscenza}

Ringrazio Mario Lombardo e il revisore anonimo per i preziosi suggerimenti su aspetti storici relativi al mondo messapico e per aver contribuito a migliorare la qualità dell'articolo.

\section{$\begin{array}{llllllllllll}\mathbf{B} & \mathbf{I} & \mathbf{B} & \mathbf{L} & \mathbf{I} & \mathbf{O} & \mathbf{G} & \mathbf{R} & \mathbf{A} & \mathbf{F} & \mathbf{I} & \mathbf{A}\end{array}$}

Ambrosini 2013: L. Ambrosini, “2. Apulia”, SE 76, 2010-2013, 305-307.

Bakkum 2009, I. G. Bakkum, The Latin Dialect of the Ager Faliscus. 150 Years of Scholarship. Part I, Amsterdam 2009.

Benelli, Marchesini e Poccetti 2019: E. Benelli, S. Marchesini e P. Poccetti (eds.), Per una definizione delle lingue e delle culture sabelliche. Defining Sabellian Languages and Cultures, Convegno Internazionale, Roma 8-9 febbraio 2018, Roma-Pisa 2019.

Blümel 1982: W. Blümel, Die aiolische Dialekte. Phonologie und Mohologie der inscriftlichen Texte aus generativer Sicht, Göttingen 1982.

Boffa 2015: G. Boffa, "Dazimos a Pitecusa” in: L. Criscuolo, G. Geraci e A. Bencivenni (ed.), Simblos. Scritti di Storia Antica 6, 2015, 7-24.

Bourdin 2012: S. Bourdin, Les peuples de l'Italie Préromaine, Rome 2012.

Ciceri 2012: M. Ciceri, "Il genitivo messapico in -ihi”, ACME 65/3, 2012, 71-102.

CIM: F. Ribezzo, Corpus Inscriptionum Messapicarum (ristampa Bari 1978 a cura di C. Santoro) [= RIGI 6, 1922; 7, 1923; 9, 1925; 10, 1926; 16, 1932; 19, 1935].

D’Andria e Lombardo 2009: F. D’Andria e M. Lombardo, "Due nuove iscrizioni messapiche da Castro”, in: F. D’Andria (a cura di), Castrum Minervae, Galatina 2009, 67-78.

Däuber 1991: U. Däuber, "L’onomastica messapica: continuità e rinnovamento ermeneutico alla luce dei nuovi dati”, in: I Messapi, Atti del Trentesimo Convegno di Studi sulla Magna Grecia, Taranto-Lecce, 4-9 ottobre 1990, Taranto 1991, 323-343.

De Simone 1964: C. de Simone, "Die messapischen Inschriften und ihre Chronologie", in: H. Krahe, Die Sprache der Illyrier, II, Wiesbaden 1964, 1-151.

De Simone 1972: C. de Simone, "La lingua messapica: tentativo di una sintesi”, in: A. Stazio (a cura di), Le genti non greche della Magna Grecia. Atti dell'XI Convegno di Studi sulla Magna Grecia. Taranto, 10-15 ott. 1971, Napoli 1972, 125-201.

De Simone 1978: C. de Simone, "Contributi per lo studio della flessione nominale messapica. Parte I: levidenza”, SE 46, 1978, 223-252. 
De Simone 1979: C. de Simone, “Onomasticon aletinum: considerazioni generali”, in: Atti del VIII convegno dei comuni Messapici, Peuceti e Dauni, Alezio, 14-15 novembre 1981, Bari 1979, 215-263.

De Simone 1982: C. de Simone, "Su tabaras (femm. -a) e la diffusione dei culti misteriosofici nella Messapia”, SE 50, 1981, 177-197.

De Simone 1988: C. de Simone, "Iscrizioni Messapiche della Grotta della Poesia (Melendugno, Lecce)”, AnnPisa, s. III, 18/2, 1988, 325-415.

De Simone 1992: C. de Simone, "Sul genitivo messapico in -ihi", AnnPisa, s. III, 22/1, 1992, 1-42.

De Simone 2012: C. de Simone, "Messapico šoranneihi ed il genitivo dei temi in -k'es $<{ }^{*}-$ kyos",

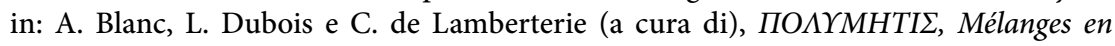
l'honneur de Françoise Bader, Leuven-Paris 2012, 315-320.

De Simone 2013: C. de Simone, "Jürgen Untermann gewidmet. 46 Jahre nach dem Erschein des Beitrages "Die Messapischen Personennamen" (1964)”, in: J. L. García Ramon, D. Kölligan e P. Poccetti (Hrsg.), Sprachkontakt und Kulturkontakt im Alten Italien: Onomastik und Lexikon. 10 Jahre nach Jürgen Untermanns "Wörterbuch des oskischUmbrischen" [Linguarum Varietas 2], 2013, 53-64.

De Simone 2017: C. de Simone, “110. Messapian”, in: J. Klein, B. Joseph e M Fritz (edd.), Handbook of Comparative and Historical Indo-European Linguistics, Berlin-Boston 2017, 1839-1850.

Deecke 1881: W. Deeke, “Zur Entzifferung der messapischen Inschriften”, RhM 36, 1881, 576-596.

Deecke 1887: W. Deeke, “Zur Entzifferung der messapischen Inschriften”, RhM 42, 1887, 226-232.

Farney e Bradley 2017: G. D. Farbey e G. Bradley (eds.), The Peoples of ancient Italy, BostonBerlin 2018.

Ferrandini Troisi 1992a: F. Ferrandini Troisi, "Epigrafi messapiche", in: L. Todisco et al., Introduzione all'artigianato della Puglia antica, Bari 1992, 214-217.

Ferrandini Troisi 1992b: F. Ferrandini Troisi, Epigrafi mobili del Museo Archeologico di Bari, Bari 1992.

Ferrandini Troisi 2005: F. Ferrandini Troisi, "Sulle tracce di Dazos", Annali della Facoltà di Lettere e Filosofia dell'Università degli Studi di Bari 48, 2005, 71-75.

Filippin c.s.: A. Filippin, "Una "lega linguistica" nell'Italia antica? Alcune considerazioni di metodo", in: E. Benelli, S. Marchesini e P. Poccetti (eds.), Per una definizione delle lingue e delle culture sabelliche. Defining Sabellian Languages and Cultures, Convegno Internazionale, Roma 8-9 febbraio 2018, Roma-Pisa in corso di stampa.

Gadaleta 2012: "Provenienze e contesti”, in: L. Todisco (ed.), La ceramica a figure rosse della Magna Grecia e della Sicilia, I-III, Roma 2012, 77-109.

Gusmani 1976a: R. Gusmani, "Messapisches”, IF 81, 1976, 143-151.

Gusmani 1976b: “Note messapiche”, in: V. Pisani e C. Santoro (ed.), Italia linguistica nuova ed antica. Studi linguistici in memoria di Oronzo Parlangèli, I, Galatina 1976, 127-145.

Gusmani 2006: R. Gusmani, "Ancora sul genitivo messapico in -(A)IHI", in: M. T. Laporta (ed.), Studi di antichità linguistiche in memoria di Ciro Santoro, Bari 2006, 199-205.

Huld 1995: M. Huld, “Grassmann's law in Messapic”, JIES 23, 1995, 147-155.

Krahe 1928: H. Krahe, "Sprachliche Untersuchungen zu den messapischen Inschriften”, Glotta $17,1 / 2,1928,81-104$.

Krahe 1929: H. Krahe, "Messapisches”, Zeitschrift für vergleichende Sprachforschung auf dem Gebiete der Indogermanischen Sprachen 56, 1/2, 1929, 133-136. 
Krahe 1955: H. Krahe, Die Sprache der Illyrier. Erster Teil. Die Quellen, Wiesbaden 1955.

Lejeune 1989: M. Lejeune, "Notes de linguistique italique, XXXIX, Génitifs en -osio et génitifs en -î", RÉL 67, 1989, 63-77.

Lejeune 1975: M. Lejeune, Lanthroponymie osque, Paris 1975.

Leumann 1972: M. Leumann, Lateinische Laut- und Formenlehre, München 1977.

Lombardo 1992: M. Lombardo (ed.), I Messapi e la Messapia nelle fonti letterarie greche e latine, Galatina 1992.

Lombardo 1994: M. Lombardo, “Tombe e riti funerari in Messapia”, Studi di Antichità 7, 1994, 25-45.

Lombardo 2013: M. Lombardo, "Cippi, iscrizioni e contesti: i rinvenimenti di Fondo Paludella nel quadro della documentazione di Vaste”, in: G. Andreassi, A. Cocchiaro e A. Dell'Aglio (ed.), Vetustis Novitatem Dare. Temi di Antichità e archeologia in ricordo di Grazia Angela Maruggi, Taranto 2013, 343-354.

Lombardo 2014: M. Lombardo, "Iapygians: The Indigenous Populations of Ancient Apulia in the Fifth and Fourth Centuries B.C.E.", in: T. H. Carpenter, K. M. Lynch e E. G. D. Robinson (eds.), The Italic People of Ancient Apulia, Cambridge 2014, 36-68.

Marchesini 1995: S. Marchesini, "Le piramidette messapiche iscritte", AnnPisa, s. III, 25/4, 1995, 1359-1385.

Marchesini 1999: S. Marchesini, “Confini e frontiera nella grecità d'Occidente: la situazione alfabetica, in: Confini e frontiera nella grecità doccidente, Atti del XXXVII convegno di Studi sulla Magna Grecia, Taranto, 3-6 ottobre 1997, Taranto 1999, 173-212.

Marchesini 2004: S. Marchesini, "Excursus metodologico sugli errori di scrittura", SCO 50, 2007, 1-58.

Marchesini 2007: S. Marchesini, Prosopographia Etrusca. II.1, Studia. Gentium Mobilitas, Roma 2007.

Marchesini 2009: S. Marchesini, Le lingue frammentarie dell'Italia Antica, Milano 2009.

Marchesini 2013a: S. Marchesini, "Quali lingue, quali popoli nell'Apulia di V e IV secolo a.C.", in: L. Todisco (ed.), La comunicazione verbale tra Greci e indigeni in Apulia nel V-IV secolo a.C. Quali elementi? Atti del Seminario di Studi linguisticim archeologici e storici. Bari, Università degli Studi 'Aldo Moro', 30 ottobre 2012, Napoli 2013, 19-33.

Marchesini 2013b: S. Marchesini, "I rapporti etrusco/retico-italici nella prima Italia alla luce dei dati linguistici: il caso della "mozione" etrusca", Rivista Storica di Antichità 43, 2013, 9-31.

Marchesini 2015: S. Marchesini, “Epigrafi messapiche nel Salento", L'Idomeneo 19, 2015, 69-78.

Marchesini c.s. 1: S. Marchesini, "The relationship between the medium and the message in the written cultures of ANCIENT ITALY: the case of Grotta Poesia", in: A. C. Cassio e S. Caszko (ed.), Multilingualism and Minority Languages in Ancient Europe. MuMiL-EU Final Congress, Roma, $26^{\text {th }}-27^{\text {th }}$ June 2019, Roma 2020.

Marchesini c.s. 2: S. Marchesini, "Ego-Inscriptions in luoghi speciali. Levidenza delle lingue dell'Italia preromana", in: I. Simon Cornago e P. Poccetti (ed.), Siste et lege. La scrittura esposta nelle società dell'Italia antica (ss. III-I a.C.), Roma, 13-14 febbraio 2020, in stampa.

Marchesini c.s. 3: S. Marchesini, "Literacy in Pre-roman Apulia. Theoretical framework and evidence", in: M. Ramirez Sánchez e N. Moncuníll (eds.), Learning Scripts, Forgetting Scripts. New approaches to the history of writing in the Roman West, Las Palmas de Gran Canaria, 8-9 novembre 2018, in stampa. 
Mastronuzzi e Ciuchini 2011: G. Mastronuzzi e P. Ciuchini, “Offerings and rituals in: a Messapian Holy Place: Vaste, Piazza Dante (Puglia, Southern Italy)”, World Archaeology, 43/4, 2011, 676-701.

Matzinger 2019: J. Matzinger, Messapisch, Wiesbaden 2019.

Mayrhofer 1986: M. Mayrhofer, Indogermanische Grammatik, Band I, Heidelberg 1986.

Meillet 1925: A. Meillet, La méthode comparative en linguistique historique, Paris 1925.

MLM: C. de Simone e S. Marchesini, Monumenta Linguae Messapicae, Wiesbaden 2012.

Mommsen 1850: T. Mommsen, Die unteritalischen Dialekte, Lipsia 1850.

Nichols 1996: J. Nichols, “The Comparative Method as Heuristic”, in: M. Durie e M. Ross (eds.), The Comparative Method Reviewed, Oxford 1996, 39-71.

NIM 1: O. Parlangèli, "Nuove Iscrizioni Messapiche (1)”, IF 70, 1965, 172-190.

NIM 2: O. Parlangèli, "Nuove Iscrizioni Messapiche (2)", Annali della Facoltà di Magistero dell'Università di Bari 6, 1966, 121-137.

NIM 3: O. Parlangèli, "Nuove iscrizioni messapiche (3)", Annali della Facoltà di Magistero dell'Università di Bari 7, 1968, 129-145.

Orioles 1972: V. Orioles, "Su alcuni fenomeni di palatalizzazione e di assibilazione nelle lingue dell'Italia antica", Studi Linguistici Salentini 5, 1972, 67-100.

Orioles 1981: V. Orioles, "Il messapico", in: E. Campanile (ed.), Nuovi materiali per la ricerca indoeuropeistica, Pisa 1981, 139-156.

Orioles 1989: V. Orioles, "Il messapico nel quadro indoeuropeo: tra innovazione e conservazione", in: E. Campanile (ed.), Rapporti linguistici e culturali tra i popoli dell'Italia antica, Pisa 1989, 157-175.

Orioles 1993: V. Orioles, “Lega linguistica italica e palatalizzazioni”, IL 16, 1993, 71-78.

PID: R. S. Conway, J. Whatmough, E. Johnson, The Prae-Italic Dialects of Italy, Cambridge/ Mass. 1933.

Pisani 1964: V. Pisani, Le lingue dell'Italia antica oltre il Latino, Torino 1964 .

Pisani 1971: V. Pisani, "La lingua dei Messapi”, Archivio Storico Pugliese 24, 1971, 229-240.

Poccetti 2008: P. Poccetti, "Un Case Study per l'identificazione di un santuario messapico: il materiale epigrafico dalla grotta di S. Maria di Agnagno (Ostuni, Brindisi)", in: X. Dupré, S. Ribichini e S. Verger (eds.), Saturnia Tellus. Definizioni dello spazio consacrato in ambiente etrusco, italico, fenicio-punico, iberico e celtico, Atti del Convegno Internazionale, Roma, 10-12 novembre 2004, Roma 2008, 233-249.

Prosdocimi 1989: A. L. Prosdocimi, "Sulla flessione nominale messapica”, AGI 74, 1989, 137-174.

Prosdocimi 1990: A. L. Prosdocimi, "Sulla flessione nominale messapica, parte seconda", AGI $75,1990,32-66$.

Prosdocimi 2006: A. L. Prosdocimi, "Il genitivo messapico in -ihi", in: R. Bombi, G. Cifoletti, F. Fusco, L. Innocentre e V. Orioles (cura di), Studi linguistici in onore di R. Gusmani, III, Alessandria 2006, 1421-1432.

Rix 1972: H. Rix, “Zum Ursprung des italischen Namensystem”, in: Aufstieg und Niedergang der römischen Welt, I.2, Berlin-New York 1972, 700-748.

Rix ST: H. Rix, Sabellische Texte: die Texte des Oskischen, Umbrischen und Südpikenischen, Heidelberg 2002 .

Ruotolo 2010: G. Ruotolo, Corpus Nummorum Rubastinorum, Bari 2010.

Santoro 1976: C. Santoro, "Nuove epigrafi messapiche (IV supplemento)", in: V. Pisani e C. Santoro, Italia Linguistica, Galatina 1976. 
Santoro 1982: C. Santoro, Nuovi studi messapici. Le epigrafi, Galatina 1982.

Santoro 1984: Nuovi studi messapici. Supplemento, Galatina 1984.

Schulze 1904: W. Schulze, Zur Geschichte der lateinischen Eigennamen, Berlin 1904 [= Neuausgabe mit einer Berichtigungsliste von Olli Salomies, Hildesheim-Zürich 1991].

Siciliano 1993: A. Siciliano, "Le zecche della Messapia", in: I Messapi, Atti del XXX Convegno di Studi sulla Magna Grecia (Taranto-Lecce 1990), Taranto 1993, 224-254.

Siciliano 2001: A. Siciliano, "La Circolazione Monetale" in: Taranto e il Mediterraneo. Atti del XLI Convegno di studi sulla Magna Grecia (Taranto 12-16 ottobre 2001), Taranto 2001, 483-517.

Solin e Salomies 1988: H. Solin e O. Salomies, Repertorium nominum gentilium et cognominum Latinorum, HIldesheim-Zürich-New York 1988.

Stazio 1971: A. Stazio, "Monetazione e circolazione monetale dell'antico Salento", in: Atti del Convegno dei comuni messapici, peuceti e dauni, Brindisi 13-15 giugno 1969, Bari 1971, 61-90.

Stazio 1972: A. Stazio, "Per una storia della monetazione dell'antica Puglia", Archivio Storico Pugliese 35, I-II, 1972, 39-47.

Tichy 1993: E. Tichy, "Kollektiva, Genus femininum und relative Chronologie im Indogermanischen", HS 106. 1, 1993, 1-19.

Todisco 2011: L. Todisco, "Mito e tragedia: ceramica figurata e comunicazione verbale nella Puglia centrale e settentrionale della seconda metà del IV secolo a.C., in: L. Todisco, Scritti di Archeologia classica. Architettura, scultura, ceramica figurata in Grecia Italia Meridionale e Sicilia, Bari 2011, 81-95.

Todisco 2012: L. Todisco, "Red-figure pottery and verbal communication in central and northern Apulia in the later fourth century BC", in: K. Bosher (ed.), Theater outside Athen. Drama in Greek Sicily and South Italy, Cambridge 2012, 251-271.

Todisco 2013: L. Todisco, “Iconografia e comunicazione verbale nei centri apuli”, in: L. Todisco (ed.), La comunicazione verbale tra Greci e indigeni in Apulia nel V-IV secolo a.C.: quali elementi? Atti del Seminario di studi linguistici, archeologici e storici, Bari 30 ottobre 2012, Napoli 2013, 35-43.

Untermann 1961: J. Untermann, Die venetischen Personennamen, Wiesbaden 1961.

Untermann 1964: J. Untermann, “Die messapischen Personennamen”, in: H. Krahe (Hrsg.), Die Sprache der Illyrier II, Wiesbaden 1964, 161-213.

Untermann 2000: J. Untermann, Wörterbuch des Oskisch-Umbrischen. Heidelberg 2000. 